\title{
General Regular Variation, Popa Groups and Quantifier Weakening
}

\author{
by \\ N. H. Bingham and A. J. Ostaszewski \\ To Harry I. Miller (22 Feb 1939 - 16/17 December 2018), man and \\ mathematician, who died with his boots on \\ 'The soldiers' music and the rites of war \\ Speak loudly for him.' (Shakespeare, Hamlet Act V.2)
}

\begin{abstract}
We introduce general regular variation, a theory of regular variation containing the existing Karamata, Bojanic-Karamata/de Haan and Beurling theories as special cases. The unifying theme is the Popa groups of our title viewed as locally compact abelian ordered topological groups, together with their Haar measure and Fourier theory. The power of this unified approach is shown by the simplification it brings to the whole area of quantifier weakening, so important in this field.
\end{abstract}

Keywords. Regular variation, general regular variation, Popa groups, Haar measure, Gołąb-Schinzel equation, Beurling-Goldie functional equation, Beurling-Goldie inequality, functional inequalities, quantifier weakening, subadditivity.

Classification: 26A03, 26A12, 33B99, 39B22.

\section{Introduction}

We recall the definition of Beurling slowly varying functions $\varphi$ (see e.g. [BinGT § 2.11], [BinO5,7]): these are positive, measurable or Baire (i.e. have the Baire property, $\mathrm{BP}$ ), are $o(x)$ at infinity (or $O(x)$, depending on context), and, with

$$
x \circ_{\varphi} t:=x+t \varphi(x)
$$

the Popa (or circle) operation or Beurling shift (§ 2 below, cf $\left(^{*}\right)$ in $\S 3$ ), satisfy

$$
\log \varphi\left(x \circ_{\varphi} t\right)-\log \varphi(x) \rightarrow 0: \quad \varphi\left(x \circ_{\varphi} t\right) / \varphi(x) \rightarrow 1 .
$$


Such $\varphi$ will play the role of auxiliary functions below. For a suitable auxiliary function $h$ and limit function $K$, called the kernel, consider also the limit relationship

$$
\left[f\left(x \circ_{\varphi} t\right)-f(x)\right] / h(x) \rightarrow K(t),
$$

where $f$ here is the function of primary interest (' $G$ for Goldie, $G$ for general': see e.g. [BinO6,10,11], [Ost4]). Specialising to $\varphi \equiv 1, h \equiv 1$ gives

$$
f(x+t)-f(x) \rightarrow K(t) \quad(K,+)
$$

('K for Karamata'). This is the defining relationship for Karamata regular variation written additively (see e.g. [BinGT Ch. 1-3]). The classical formulation $-(K, \times)$ of $\S 3$ - used by Karamata worked multiplicatively [BinGT, Ch. 1]. One needs to be able to pass between these two, using the familiar exp-log isomorphism between the additive group of reals (Haar measure Lebesgue measure $\mathrm{d} x$ or $\mathrm{d} \lambda(x))$ and the multiplicative group of positive reals (Haar measure $\mathrm{d} x / x$ ). Specialising instead to $\varphi \equiv 1, h$ slowly varying (in Karamata's, multiplicative, sense: [BinGT, Ch. 1]) gives

$$
[f(x+t)-f(x)] / h(x) \rightarrow K(t),
$$

the defining relationship for Bojanic-Karamata/de Haan regular variation [BinGT, Ch. 3], while specialising to $f=\log \varphi, h \equiv 1, K=0$ gives Beurling slow variation as above. We call the limit relationship $(G)$ above general regular variation, as it contains the other three. We give a unified treatment, using the algebraicization provided by the Popa groups of $\S 2$ below.

The kernel functions $K$ are characterized by the functional equations which they satisfy. The classical Karamata setting yields the multiplicative Cauchy functional equation, $(C F E)$ for short below [Kuc]. Here we will need, among others, the Chudziak-Jabłonska functional equation

$$
K\left(u \circ_{\eta} v\right)=K(v) K(u),
$$

and its generalizations, studied in $\S 3$ below.

As usual (see e.g. [BinO1,9]), we pass between the measurable and Baire cases (in any form of regular variation) bitopologically - by passing between the Euclidean and density topologies. The same will be true in the Popa groups below, which are isomorphic to the reals algebraically and bitopologically; we thus extend the terms Euclidean and density topologies to these Popa isomorphs also.

In what follows, we occasionally refer to the extended version of this paper at arXiv: 1901.05996. 


\section{Popa groups}

Above we have used the Popa operation as a simplifying notational device for the regular variation above (general or otherwise), involving limits as $x \rightarrow \infty$. But its usefulness is far greater, and is not confined to limits, as emerged in [BinO7], [Ost3]. Here one allows other auxiliary functions $h$, with corresponding circle operations $\circ_{h}$. This is most useful when the circle operation is associative, and this requires $h$ to satisfy the Gotab-Schinzel functional equation:

$$
h\left(s \circ_{h} t\right)=h(s+h(s) t)=h(s) h(t)
$$

(cf. [Jav]). Thus $(G S)$ expresses homomorphy in this context, which will occur in the regular-variation context after the passage to the limit $x \rightarrow \infty$. Indeed, such an $h$ generates group structures on subsets of $\mathbb{R}$, that are in fact isomorphic to the group $\left(\mathbb{R}_{+}, \times\right)$, where $\mathbb{R}_{+}:=(0, \infty)$. It is to these Popa groups $[\mathrm{Pop}]$ that we now turn.

Orientational convention. Parameters $\rho<0$ would give Popa groups $\mathbb{G}_{\rho}$ (below) as $(-\infty,-1 / \rho)$. To conform to usage in the motivating area, regular variation, in which one approaches $+\infty$ rather than $-\infty$, we disregard parameters $\rho<0$, so that $\mathbb{R}_{+} \subseteq \mathbb{G}_{\rho}^{+}$(below).

Write $G S$ for the set of positive solutions $h$ of $(G S)$. It emerges that, being thus bounded below, they are continuous, and of the form

$$
\eta(t)=\eta_{\rho}(t):=1+\rho t
$$

for $t>-1 / \rho$, with the parameter $\rho \geq 0$; for a proof see [Brz2] and [BrzM], or the more direct [Ost5, §5] (see also [AczD] and the surveys [Brz1] and [Jab1,3]; cf. [Ost3]). For a discussion of circumstances when local boundedness implies the continuity of solutions, for the family relevant here of functional equations related to $(G S)$, see [Jab2]. For $\eta \in G S$, put

$$
\mathbb{G}_{\eta}^{*}:=\{x \in \mathbb{R}: \eta(x) \neq 0\}
$$

equipped with $\circ_{\eta}$, this is a group. When $\eta=\eta_{\rho}$ this operation is given explicitly by

$$
x \circ_{\rho} y=x+y(1+\rho x) \text {, }
$$


so that, adapting notation, $\mathbb{G}_{\rho}^{*}:=\left\{x \in \mathbb{R}: x \neq \rho^{*}\right\}$, where $\rho^{*}:=-1 / \rho$, the Popa centre.

The operation $\circ_{\rho}$ may also be rendered by reference to the equation $(G S)$ in the current context:

$$
\eta_{\rho}\left(x \circ_{\rho} y\right)=\eta_{\rho}(x) \eta_{\rho}(y) \quad\left(x, y \in \mathbb{G}_{\rho}^{*}\right),
$$

and thereby to the underlying role of the multiplicative positive reals $\mathbb{R}_{+}$. Here one has the conjugacy

$$
x \circ_{\rho} y=\eta_{\rho}^{-1}\left(\eta_{\rho}(x) \eta_{\rho}(y)\right)=[(1+\rho x)(1+\rho y)-1] / \rho \quad \text { (conj) }
$$

(which gives for $\rho=1$ the circle operation of ring theory: cf. [Jac, II.3], [Coh, 3.1], and [Ost4, §2.1] for the historical background). It emerges from here that the following subgroups of $\mathbb{G}_{\eta}^{*}$ are of greater significance:

$$
\mathbb{G}_{\eta}:=\{x \in \mathbb{R}: \eta(x)>0\}: \quad \mathbb{G}_{\rho}:=\{x \in \mathbb{R}: 1+\rho x>0\},
$$

by virtue of being isomorphic with $\left(\mathbb{R}_{+}, \times\right)$when $\rho>0$. (Likewise, the groups $\mathbb{G}_{\rho}^{*}$ are all isomorphic with $\left(\mathbb{R}^{*}, \times\right)$, with $\mathbb{R}^{*}:=\mathbb{R} \backslash\{0\}$.)

For $\rho \downarrow 0$ we interpret $\rho^{*}$ to mean $-\infty$; likewise $\rho^{*}$ is to mean 0 in the limit as $\rho \rightarrow+\infty$. Then the group $\left(\mathbb{R}_{+}, \times\right)$may be viewed conveniently as $\mathbb{G}_{\infty}$, since scaling $x \mapsto x / \rho$ for $\rho>0$ establishes as isomorphic the group structure on $\mathbb{R}_{+}$conferred by the shifted product (cf. [Ost4, Prop. C ' $b$-shifted' sum]):

$$
x \times_{\rho} y:=\rho x y: \quad(x \times y) / \rho=(x / \rho) \times_{\rho}(y / \rho),
$$

so that by (conj) above

$$
x \circ_{\rho} y \sim \rho x y=x \times_{\rho} y \quad(\rho \rightarrow+\infty) .
$$

More accurately, we should see $\mathbb{G}_{\infty}=\mathbb{R}_{+}$as the rescaled limit of $\mathbb{G}_{\rho}$ as $\rho \rightarrow \infty$, as follows:

$$
\left(x \circ_{\rho} y\right) / \rho=x / \rho+y / \rho+x y \rightarrow x y, \text { as } \rho \rightarrow \infty .
$$

So we write $\circ_{\infty}$ as ordinary multiplication:

$$
x \circ_{\infty} y:=x \times y=x y .
$$

We return to this matter below after Theorem 2 (reviewing the full spectrum of Popa groups) and again in Theorem 3. 
For later use in Theorem 1 below, note that the domain of $\mathbb{G}_{\rho}$ for each $\rho \in[0, \infty]$ is an open set in $\mathbb{R}$ and the two partial derivatives of the group-law function

$$
F_{\rho}(x, y):=x \circ_{\rho} y \quad(\rho \in[0, \infty])
$$

with domain the subset $\mathbb{G}_{\rho} \times \mathbb{G}_{\rho} \subseteq \mathbb{R} \times \mathbb{R}$ are continuous under the Euclidean topology; indeed, $\partial F / \partial x=1+\rho y=\eta_{\rho}(y)$ (or $y$, when $\rho=\infty$ ) independent of $x$, and similarly for $\partial F / \partial y$. The Jacobian of the $t$-shift $x \mapsto t \circ_{\rho} x$ is $\eta_{\rho}(t)$ and is independent of $x$.

We note that one has $1_{\mathbb{G}}=0$ for $\mathbb{G}=\mathbb{G}_{\rho}$ except for $\mathbb{G}=\mathbb{G}_{\infty}$, when $1_{\mathbb{G}}=$ 1. The inverse of $t$ in $\mathbb{G}_{\eta}$ will variously be denoted by $t_{\eta}^{-1}$ or $t_{\rho}^{-1}$ (including $t_{\infty}^{-1}=1 / t$ for $t>0$ ), or ${ }_{\rho} t$, as convenience may dictate; here

$$
t_{\rho}^{-1}=-t / \eta_{\rho}(t), \text { for } \rho \in[0, \infty) .
$$

We will also need to designate location in $\mathbb{G}_{\rho}$ to either side of $1_{\mathbb{G}}$, using the notation

$$
\mathbb{G}_{\rho}^{+}:=\left\{x \in \mathbb{G}_{\rho}: x>0 \& 1+\rho x>0\right\}=\mathbb{R}_{+}=(0, \infty), \text { for } \rho \in[0, \infty),
$$

with

$$
\mathbb{G}_{\infty}^{+}:=\left\{x \in \mathbb{R}_{+}: x>1\right\}=(1, \infty)
$$

and

$$
\mathbb{G}_{\rho}^{-}:=\left\{x \in \mathbb{G}_{\rho}: x<0 \& 1+\rho x>0\right\}=(-1 / \rho, 0), \text { for } \rho \in[0, \infty),
$$

with

$$
\mathbb{G}_{\infty}^{-}:=(0,1), \text { for } \rho=\infty .
$$

Now (conj) above demonstrates that $\circ_{\rho}$ may be expressed in terms of the ring operations of $\mathbb{R}$, and so permits other features of $\mathbb{R}$ to be imported into $\mathbb{G}_{\rho}$. There are several possibilities here. The Popa groups may inherit either of the two canonical topological structures of their isomorphs, again enabling bitopological passage between them (as in $\S 1$ ). Thus they inherit a Euclidean topology, from which they derive their own metric structures; this is generated by (open) intervals (see Prop. 2 below), and makes $\mathbb{G}_{\rho}$ a locally compact abelian topological group. In turn this allows reference to Haar measure, and so to the second possibility: the Haar-density topology of $\mathbb{G}_{\rho}$, which agrees with the topology induced on $\mathbb{G}_{\rho}$ by the (Lebesgue) density 
topology on $\mathbb{R}$ (corresponding to Lebesgue measure $\lambda$ ) and with the Haardensity topology of $\mathbb{R}_{+}$. In particular, the two topologies make available as a tool the interior-point theorem of Steinhaus-Weil from measure theory [Ste] [Wei], and the Piccard-Pettis category analogue [Pic] [Pet] (cf. [BinO13]). Before identifying the (normalized) Haar measure of $\mathbb{G}=\mathbb{G}_{\rho}$, written $\eta_{\mathbb{G}}$, we observe in Prop. 2 below that $\mathbb{G}$ has a natural order which coincides with the usual order on $\mathbb{R}$. We also identify the associated canonical invariant metrics on $\mathbb{G}$, below.

We recall that by the Birkhoff-Kakutani Theorem ([Bir], [Kak1]; cf. [DieS, $\S 3.3, \S 8]$ ) we may equip any metrizable group $G$ with a (left-)invariant metric $d_{G}^{L}$ (i.e. with $d_{G}^{L}(g h, g k)=d_{G}^{L}(h, k)$, for all $\left.g, h, k \in G\right)$, equivalently with a (group) norm $\|g\|:=d_{G}^{L}\left(g, 1_{G}\right)$, as in [BinO2] ('pre-norm' in [ArhT]), that generates the topology. Its defining features are:

(i) $\|g\|=0$ iff $g=1_{G} \quad$ (positive definiteness);

(ii) $\|g h\| \leq\|g\|+\|h\|$ (triangle inequality);

(iii) $\left\|g^{-1}\right\|=\|g\| \quad$ (symmetry).

Group norms, like Haar measure, are determined to within a positive scale factor. In Proposition 1 below, we choose the normalizing factor there so as to unify treatment of the three cases $\rho=0, \rho \in(0, \infty)$, and $\rho=\infty$.

Proposition 1. (a) A group norm on $\mathbb{G}_{\rho}$ for $\rho \in[0, \infty)$ is given by

$$
\|t\|_{\rho}:=\left\{\begin{array}{cl}
|\log (1+\rho t)|(1+\rho) / \rho, & \text { if } \rho>0 \\
|t|, & \text { if } \rho=0 .
\end{array}\right.
$$

(b) In particular, $\|1\|_{\rho}=(1+\rho)[\log (1+\rho)] / \rho$, and $\|t\|_{\rho} \rightarrow|t|$ as $\rho \rightarrow 0$ (for $t \neq 0$ ).

(c) A group norm on $\mathbb{G}_{\infty}=\mathbb{R}_{+}$is given by

$$
\|t\|_{\infty}:=|\log t|=\lim _{\rho \rightarrow \infty} d_{\rho}(1, t)=\lim _{\rho \rightarrow \infty}\left\|1 \circ t_{\rho}^{-1}\right\|_{\rho} .
$$

Proof. (a) Here property (i) of the norm is clear; as for (ii), we have

$$
\left\|s 0_{\rho} t\right\|_{\rho}=|\log (1+\rho(s+t+\rho s t))|(1+\rho) / \rho=|\log (1+\rho s)(1+\rho t)|(1+\rho) / \rho \leq\|s\|_{\rho}+\|t\|_{\rho} .
$$

Then (iii) follows, since $\eta_{\rho}\left(t_{\rho}^{-1}\right)=\eta_{\rho}(t)^{-1}$ i.e. with $s=t_{\rho}^{-1}$

$$
(1+\rho s)=1 /(1+\rho t)
$$


(or, from (conj) above, $1_{\rho}=0=s \circ_{\rho} t=[(1+\rho s)(1+\rho t)-1] / \rho$, so $(1+$ $\rho s)(1+\rho t)=1)$.

(b) The second assertion follows by L'Hospital's rule (or as $\log (1+\rho t) \sim \rho t$ as $\rho \downarrow 0)$.

(c) The first assertion is similar to but simpler than in (a), and the second follows from (a) and (inv), as $\left\|1 \circ_{\rho} t_{\rho}^{-1}\right\|_{\rho}=|\log [(1+\rho) /(1+\rho t)]|(1+\rho) / \rho$.

Remark. The inclusion of the scaling factor $(1+\rho)$ is dictated by Haarmeasure normalization concerns, below.

Proposition 2. For $\rho \in[0, \infty)$, the set $[0, \infty)$ is a sub-semigroup of $\mathbb{G}_{\rho}$; the induced weak order, $y \leq_{\rho} x$ iff $x \circ_{\rho} y^{-1} \in[0, \infty)$, coincides with $y \leq x$, and similarly for the strong order, $y<_{\rho} x$ iff $x \circ_{\rho} y^{-1} \in(0, \infty)$.

Furthermore, for $c \in \mathbb{G}_{\rho}$ and $a<b$,

$$
a \circ_{\rho} c<b \circ_{\rho} c ;
$$

in particular, for the interval $(a, b)$,

$$
(a, b) \circ_{\rho} c=\left(a \circ_{\rho} c, b \circ_{\rho} c\right):
$$

the Euclidean topology on $\mathbb{G}_{\rho}$ is invariant under translation by $\circ_{\rho}$. Likewise, for $\rho>0$, if $a<b$ and $c<d$, with $a, b, c, d \in \mathbb{G}_{\rho}$,

$$
a \circ_{\rho} c<b \circ_{\rho} d
$$

and likewise for weak inequality, so that

$$
s \leq t \text { iff } s_{\rho}^{-1} \geq t_{\rho}^{-1} \quad\left(s, t \in \mathbb{G}_{\rho}\right) .
$$

Proof. For the first assertion observe that

$0 \leq x-(1+\rho x) y /(1+\rho y)$ iff $0 \leq x(1+\rho y)-(1+\rho x) y=x-y$, as $1+\rho y>0$.

Similarly, since $1+\rho c>0$, if $a<b$, then

$$
a \circ_{\rho} c=c+a(1+\rho c)<c+b(1+\rho c)=b \circ_{\rho} c .
$$

From here, as $a \leq b$ and $c \leq d$,

$$
a \circ_{\rho} c \leq b \circ_{\rho} c \text { and } c \circ_{\rho} b<d \circ_{\rho} b: \quad a \circ_{\rho} c \leq b \circ_{\rho} d .
$$


Finally, $s \leq t$ iff

$$
-1 /(1+\rho t) \geq-1 /(1+\rho s): \quad 1-1 /(1+\rho t) \geq 1-1 /(1+\rho s): \quad-\rho t_{\rho}^{-1} \geq-\rho s_{\rho}^{-1} .
$$

Remarks. 1. Proposition 2 above clarifies both our earlier orientation convention and the convention adopted in [Ost4, §3.2] and followed here (especially in $\S 3$ Prop. 4): that a real-valued function on $\mathbb{G}_{\rho}$ is termed positive if it is positive on the sub-semigroup $\left(1_{\rho}, \infty\right)$ - see $\S 4$.

2. It now follows that under the order topology $\mathbb{G}_{\rho}$ is an open subspace of $\mathbb{R}$ (i.e. embeddable in $\mathbb{R}$ in the sense below) and likewise the partial derivatives of the group-law function $F_{\rho}(x, y)$ (above) are continuous on $\mathbb{G}_{\rho} \times \mathbb{G}_{\rho} \subseteq$ $\mathbb{R} \times \mathbb{R}$, cf. comments after the display (resc).

Definitions 1. Say that a locally compact abelian group $(\mathbb{G}, \circ)$ is embeddable in $\mathbb{R}$, if $\mathbb{G}$ is an open subspace of $\mathbb{R}$.

2. For $(\mathbb{G}, \circ)$ a locally compact abelian group embeddable in $\mathbb{R}$, say that the group action has relative invariance (i.e. relative to $\mathbb{G}$, cf. [HewR, 15.18]) if for any open $U$ around $1_{\mathbb{G}}$ such that $g \circ U$ is a neighbourhood of $g \in \mathbb{G}$,

$$
\lambda(g \circ U)=\chi(g) \lambda(U)
$$

with ( $\lambda$ Lebesgue measure and) $\chi: \mathbb{G} \rightarrow \mathbb{R}_{+}$.

Observation. Here $\chi$ is a continuous homomorphism:

$$
\chi(g \circ h)=\chi(g) \chi(h) .
$$

Indeed

$$
\lambda(g \circ h \circ U)=\chi(g \circ h) \lambda(U)=\chi(g) \lambda(h \circ U)=\chi(g) \chi(h) \lambda(U) .
$$

Furthermore, for $U \subseteq \mathbb{G}$ non-empty, open with compact closure, the map $g \mapsto \lambda(g \circ U) / \lambda(U)$ is continuous in $g$, since $\lambda$ is translation-invariant and the group operation $\circ$ is continuous on an open subset of $\mathbb{R}$. This relates to the notion of mobility of a measure, for which see [BinO13] and literature cited there. Evidently, $1 / \chi$ is proportional to the Radon-Nikodym derivative of the restriction measure $\lambda \mid \mathbb{G}$ w.r.t. $\lambda$ and so also of any Haar measure on $\mathbb{G}$. 
Examples: 1. For $(\mathbb{G}, \circ)=\left(\mathbb{R}_{+}, \times\right), \chi(g)=g$, since for $g>0$ and $a<b$

$$
\lambda(g \times(a, b))=\lambda(g a, g b)=g(b-a) .
$$

2. For $(\mathbb{G}, \circ)=\mathbb{G}_{\rho}$ with $\rho \in[0, \infty)$,

$$
g \circ_{\rho}(a, b)=g+\eta_{\rho}(g)(a, b): \quad \lambda\left(g \circ_{\rho}(a, b)\right)=\eta_{\rho}(g) \lambda(a, b) .
$$

So here $\chi(g)=\eta_{\rho}(g)=1+\rho g$.

3. The Beurling $t$-shift $x \mapsto t \circ_{\varphi} x=t+x \varphi(t)$, for $\varphi \in S E$, gives 'asymptotic relative invariance' with $\chi=\varphi$ : see the arXiv version.

Theorem 1 (Haar measure). Normalized Haar measure on the Popa group $\mathbb{G}=\mathbb{G}_{\rho}$, with the Euclidean topology giving the interval $(0,1)$ measure $\|1\|_{\rho}$ for $\rho \geq 0$, has Radon-Nikodym derivative $(1+\rho) / \eta_{\rho}(g)$ w.r.t. $\mathrm{d} g$, the Lebesgue measure on the additive reals, that is

$$
\begin{aligned}
\mathrm{d} \eta_{\mathbb{G}}(t) & =(1+\rho) \mathrm{d} t / \eta(t)=(1+\rho) \mathrm{d} t / \eta_{\rho}(t) \\
& =(1+\rho) \mathrm{d} t /(1+\rho t), \text { for } \eta=\eta_{\rho}
\end{aligned}
$$

In particular, as $1_{\rho}=0$, the group norm satisfies

$$
\|x\|_{\rho}=\left|\eta_{\mathbb{G}}\left(\left(1_{\rho}, x\right)\right)\right|=\left|\int_{0}^{x}(1+\rho) \mathrm{d} t /(1+\rho t)\right|=\frac{1+\rho}{\rho}|\log (1+\rho x)| .
$$

Proof. This result follows immediately from [HewR, $§ 15.17(\mathrm{e})]$ and Remark 2 above.

See the arXiv version of the paper for an alternative more direct proof, more closely aligned to relative invariance, needed later in the preamble to Theorem 4, yielding Haar measure proportional to

$$
\mathrm{d} t / \chi(t)
$$

So for the normalized measure $\eta_{\mathbb{G}}$ of Theorem 1 , the Radon-Nikodym derivative at $g$ is proportional to $1 / \eta_{\rho}(g)$. The proportionality constant $(1+\rho)$ allows for the two extreme $\rho$ values, yielding Lebesgue measure $\mathrm{d} t$ on the additive reals for $\rho=0$, and Haar measure $\mathrm{d} t / t$ on the multiplicative reals $\mathbb{R}_{+}$as $\rho \rightarrow \infty$ :

$$
\mathrm{d} \eta_{\mathbb{G}}(t)=\frac{1+\rho}{1+\rho t} \mathrm{~d} t \rightarrow \mathrm{d} t \text { as } \rho \rightarrow 0, \quad \rightarrow \frac{\mathrm{d} t}{t} \text { as } \rho \rightarrow \infty .
$$


The completed spectrum of Popa groups. Up to scaling, there are just three Popa operations/groups, corresponding to $\rho=0,1, \infty$, namely $+, \circ, \times$ with $\circ$ the circle operation of ring theory as above; indeed the scaling map $x \mapsto x / \rho$ is an isomorphic stretching for $\rho \in(0,1]$, or shrinking for $\rho \in[1, \infty)$, of $\mathbb{G}_{1}$ onto $\mathbb{G}_{\rho}$. The entire family forms a continuous system, termed by Braconnier [Bra] a spectrum, with embeddings (bonding maps) given here by $e_{\rho}^{\sigma}: x \mapsto x \sigma / \rho$ of $\mathbb{G}_{\sigma}$ onto $\mathbb{G}_{\rho}$. These are compatible under composition:

$$
e_{\rho}^{\tau}(x)=e_{\rho}^{\sigma} \circ e_{\sigma}^{\tau}(x)=\frac{\sigma}{\rho} \frac{\tau}{\sigma} x=\frac{\tau}{\rho} x,
$$

and so the ordered system $\left\{e_{\rho}^{\sigma}: 0<\rho \leq \sigma<\infty\right\}$ gives rise to an inductive limit group as $\rho \rightarrow \infty$ :

$$
\mathbb{G}_{\infty}:=\lim _{\longrightarrow} \mathbb{G}_{\rho} .
$$

For their topological properties see the equivalent (dual) inverse systems of [Eng, §2.5]; compare [ArhT, §10.1], where the spectra are well-ordered. (Recall the elements of this limit are 'threads', i.e. sequences $\mathbf{x}=\left(x_{\sigma}: 0<\right.$ $\sigma<\infty)$ with compatible co-ordinates, so that here $x_{\rho}=x_{\sigma} \sigma / \rho$ for $\sigma<\rho$; the group product $\mathbf{x y}$ here is $(\mathbf{x y})_{\rho}=x_{\rho} \circ_{\rho} y_{\rho}$.) As members of the family are mutually isomorphic, so is the limit; interpreting that to be $\mathbb{R}_{+}$promotes $\eta_{\rho}$ to the role of the limiting embedding of $\mathbb{G}_{\rho}$ into $\mathbb{G}_{\infty}$. Above, since the embeddings are isomorphisms, one may reverse the order and also take limits as $\rho \rightarrow 0$, yielding

$$
\mathbb{G}_{0}:=\lim _{\longleftarrow} \mathbb{G}_{\rho} .
$$

Remarks. 1. The discontinuity in $1_{\rho}$ at $\rho=\infty$ may be avoided, using an appropriate ' $r$-shifted product' (with $r=r(\rho)$ ); for this, see the arXiv version.

2. The alternative normalization of Haar measure is $\delta\left(1_{\rho}\right)=1$, as

$$
x \circ_{\rho} y=x+y+\rho x y \sim x+y \quad(x, y \rightarrow 0) .
$$

We use instead $\delta\left(1_{\rho}\right)=1+\rho$, as this includes $\rho=\infty$ also.

3. In connection with uniqueness (for given growth rate) of (regular) solutions of $(C F E)$, note that for $\rho \in[0, \infty]$ the group $\mathbb{G}_{\rho}$ has a unique idempotent, $c=0$ (replaced by $c=1$ in the case $\rho=\infty$ ):

$$
\begin{aligned}
& \rho \in[0, \infty): \quad c=c \circ_{\rho} c: \quad 0=c+\rho c^{2}=c(1+\rho c): \quad c=0(\text { as } 1+\rho c>0) . \\
& \rho=\infty: \quad c=c^{2}: \quad c=1 \text { in } \mathbb{R}_{+}=(0, \infty) .
\end{aligned}
$$


4. The origin of the Haar measures $\mathrm{d} t, \mathrm{~d} t / t$ for the cases $\rho=0, \infty$ above are clear: the arithmetic operations + and $\times$. From the canonical $\mathrm{d} t / t$ case one may infer the general $\rho \in(0, \infty)$ case by a change of origin to the Popa centre $-1 / \rho$. That of the intermediate values $\rho \in(0, \infty)$ is exemplified by the case $\rho=1$, giving $\mathrm{d} t /(1+t)$. This arises via the role of the Beck sequences in the proof of Theorem 4 and the Remark below it, and is an instance of the ergodic theorem (see e.g. Billingsley [Bil, Ch. $1 \S 4$ ] and Remark 4 below). The same measure arises in the Gauss-Kuzmin theorem on continued fractions, and for the same reason - see $\S 6.2$ below and again [Bil, Ch. $1 \S 4$ ].

5. As just mentioned above: see [BinO7, Prop. 11 (iv)] for the sense in which the Beck sequence of iterates grows arithmetically, which links their averages with the arithmetic means in the (Birkhoff-Khinchin) ergodic theorem.

6. Formal group laws $F(X, Y)$ refer to an $F$ that is a polynomial series in two indeterminates over a ring ('formal' here signals potential absence of series convergence) capable of generating a group structure with 0 its neutral element (along the lines of $F(X, F(Y, Z))=F(F(X, Y), Z)$ ). These include the important example of the multiplicative formal group law $F(X, Y)=$ $X+Y+X Y$. This is 1-dimensional: more generally $X$ and $Y$ may also denote $n$-dimensional vectors of indeterminates. These provide a tool for number theory, arithmetical algebraic geometry, algebraic geometry, and algebraic topology; for a textbook account see [Haz].

7. The limiting behaviour of the moving average $\left[U\left(x \circ_{\varphi} t\right)-U(x)\right] / \varphi(x)$ of $U$ and the Tauberian one-sided conditions studied by Bingham and Goldie [BinG2] emerge in $\S 3$ below directly from the asymptotic operator $K_{h}^{\varphi}(t, x)$ with the specialization $h=\varphi$. The group norms exhibited in Th. 1 above thus coincide with the measures of occupation 'times' (on $\left[1_{\rho}, x\right]$ ) of the associated limiting velocity flow $\mathrm{d} w(t) / \mathrm{d} t=\eta_{\rho}(w(t))$ (for $\eta_{\rho}=\eta^{\varphi}$ in the sense of $\S 3$ below): see $[\mathrm{BinO} 7, \S 2]$. Here Lebesgue measure $\mathrm{d} t$ measures time, and equates with $w^{\prime}(t) \cdot \mathrm{d} t / \eta(w(t))$, i.e. the Haar integral of the flow rate.

We recall that the dual $\hat{G}$ of a locally compact abelian group $G$ comprises the characters, the continuous homomorphisms from $G$ to $\mathbb{T}$, the unit circle in the complex plane $\mathbb{C}$. For $\eta_{G}$ a Haar measure on $G$, the Fourier transform (see [Tit], [Wie] for the classical setting) is defined by

$$
\hat{f}(\gamma)=\mathcal{F}_{G}\{f\}(\gamma):=\int_{G} f(g) \gamma(-g) \mathrm{d} \eta_{G}(g) \quad(\gamma \in \hat{G})
$$

for background on locally compact abelian groups, see [Rud], [Loo]. We spe- 
cialize this in Theorem 2 below to the Popa group $\left(\mathbb{G}_{\rho}, \circ_{\rho}\right)$ for $0<\rho<\infty$. It is helpful to first consider the extreme cases $\rho=0$ and $\rho=\infty$, corresponding respectively to the familiar cases $G=(\mathbb{R},+)$ and $G=\left(\mathbb{R}_{+}, \times\right)$. In the first case $\hat{G}=G=(\mathbb{R},+)[$ Loo, $35 \mathrm{C}]$, and we may write for $\gamma \in \mathbb{R}$

$$
\gamma(w)=e^{i \gamma w} \quad(w \in \mathbb{R}),
$$

so that, for $f \in L^{1}(\mathbb{R})$,

$$
\hat{f}(\gamma)=\int_{\mathbb{R}} f(w) e^{-i \gamma w} \mathrm{~d} w \quad(\gamma \in \mathbb{R}) .
$$

We pass to the second case via the isomorphism $w=\log v$ (for $v \in \mathbb{R}_{+}$) which, for $f \in L^{1}\left(\mathbb{R}_{+}\right)$, yields both the Fourier transform and the Mellin transform

$$
\hat{f}(\gamma)=\int_{0}^{\infty} f(v) e^{-i \gamma \log v} \mathrm{~d} v / v \quad \check{f}(\gamma)=\int_{0}^{\infty} f(t) t^{-\gamma} \mathrm{d} t / t \quad(\gamma \in \mathbb{R}),
$$

with characters in the latter case written multiplicatively as $\gamma(t)=t^{\gamma}$.

We now specialize from a locally compact group to the Popa group $\mathbb{G}_{\rho}$ for $\rho>0$. As we shall see, the Fourier-Popa transform of $f: \mathbb{G}_{\rho} \rightarrow \mathbb{R}$ is in fact the ordinary Fourier transform of an affinely related function $f_{\rho}: \mathbb{R}_{+} \rightarrow \mathbb{R}$ :

$$
f_{\rho}(t)=\frac{1+\rho}{\rho} f\left(\eta_{\rho}^{-1}(t)\right)=\frac{1+\rho}{\rho} f((t-1) / \rho) \quad(\rho \in(0, \infty)) \quad\left(t \in \mathbb{R}_{+}\right) .
$$

This includes the case $\rho=\infty$, after rescaling: $f_{\rho}(\rho t) \mapsto f(t)$ (cf. $\S 2$ for this rescaling).

Theorem 2 (Fourier transform). For the Popa group $G=\left(\mathbb{G}_{\rho}, \circ_{\rho}\right)$ with $0<\rho<\infty$, the characters $\gamma \in \hat{G}$ are

$\gamma(u):=e^{i \gamma \log (1+\rho u)} \quad\left(\gamma \in \mathbb{R}, u \in \mathbb{G}_{\rho}\right), \quad$ so of the form $e^{i \gamma \operatorname{sign}(u) \cdot\|u\|}$ for $u \in \mathbb{G}_{\rho}^{ \pm}$.

So, writing $+_{\rho}$ and $-{ }_{\rho}$ for the operations of $\circ_{\rho}$ and its inverse here, the Fourier transform corresponding to the canonical Haar measure of Theorem 1 is

$$
\begin{aligned}
\hat{f}(\gamma) & =\int_{\mathbb{G}_{\rho}} f(u) \gamma\left(-{ }_{\rho} u\right)(1+\rho) \mathrm{d} u /(1+\rho u)=\int_{0}^{\infty} f_{\rho}(t) e^{i \log t^{-\gamma}} \mathrm{d} t / t \\
& =\int_{0}^{\infty} f((t-1) / \rho) e^{i \log t^{-\gamma}} \mathrm{d} t / t .
\end{aligned}
$$


The corresponding Mellin transform is thus

$\check{f}(\gamma)=\int_{0}^{\infty} f_{\rho}(t) t^{-\gamma} \mathrm{d} t / t=\int_{0}^{\infty} f_{\rho}(1 / u) u^{\gamma} \mathrm{d} u / u=\int_{0}^{\infty} f((1-u) /(\rho u)) u^{\gamma} \mathrm{d} u / u$.

In summary: for $0<\rho<\infty$ and $f \in L^{1}\left(\mathbb{G}_{\rho}\right)$

$$
\mathcal{F}_{\mathbb{G}_{\rho}}\{f\}(\gamma)=\mathcal{F}_{\mathbb{R}_{+}}\left\{f_{\rho}\right\}(\gamma) \quad(\gamma \in \mathbb{R}),
$$

the limiting shift relations being:

$$
\begin{array}{rlr}
\lim _{\rho \rightarrow \infty} \mathcal{F}_{\mathbb{R}_{+}}\left\{f_{\rho}(\rho \cdot)\right\}(\gamma) & \rightarrow \mathcal{F}_{\mathbb{R}_{+}}\{f\}(\gamma) & (\gamma \in \mathbb{R}), \\
\lim _{\rho \downarrow 0} \mathcal{F}_{\mathbb{G}_{\rho}}\{f\}(\gamma / \rho) & \rightarrow \mathcal{F}_{\mathbb{R}}\{f\}(\gamma) \quad(\gamma \in \mathbb{R}) .
\end{array}
$$

Proof. Applying the isomorphisms $\eta_{\rho}:\left(\mathbb{G}_{\rho}, \circ\right) \rightarrow\left(\mathbb{R}_{+}, \times\right)$and $\log :$ $\left(\mathbb{R}_{+}, \times\right) \rightarrow(\mathbb{R},+)$ and using $u, v, w$ as corresponding generic group elements with $w=\log v$ and $v=1+\rho u$, the character representation for $(\mathbb{R},+)$ recalled above gives the character representation for $\left(\mathbb{G}_{\rho}, 0\right)$ as asserted. By (inv) above

$$
1+\rho\left(-{ }_{\rho} u\right)=1 /(1+\rho u),
$$

so substitution for $\gamma\left(-{ }_{\rho} u\right)$ gives the Fourier transform as

$\hat{f}(\gamma)=\int_{\mathbb{G}_{\rho}} f(u) \gamma\left(-{ }_{\rho} u\right)(1+\rho) \mathrm{d} u /(1+\rho u)=\int_{-1 / \rho}^{\infty} f(u) e^{-i \gamma \log (1+\rho u)}(1+\rho) \mathrm{d} u /(1+\rho u)$.

Putting $t=\eta_{\rho}(u)=1+\rho u$ gives

$$
\hat{f}(\gamma)=\int_{0}^{\infty} f_{\rho}(t) e^{i \log t^{-\gamma}} \mathrm{d} t / t
$$

This gives the first form of the Mellin transform above; for the second, take $u=1 / t$.

For the final assertion with $f \in L^{1}\left(\mathbb{G}_{\rho}\right)$, in the limit as $\rho \downarrow 0$ (since here again $\log (1+\rho u) / \rho \rightarrow u)$ we obtain the usual transform on $\mathbb{R}$

$\hat{f}(\gamma / \rho)=\int_{-1 / \rho}^{\infty} f(u) e^{-i(\gamma / \rho) \log (1+\rho u)}(1+\rho) \mathrm{d} u /(1+\rho u) \rightarrow \int_{-\infty}^{\infty} f(u) e^{-i \gamma u} \mathrm{~d} u$,

the convergence being dominated.

The Fourier and Mellin transforms above may be extendable from real to complex arguments by analytic continuation; when possible, this is often useful. For background, see the classic by Paley and Wiener [PalW]; for applications see e.g. [BinGT, Ch. 4, 5]. 


\section{Asymptotic actions and functional equa- tions}

We begin with the Karamata asymptotic operator $K$ acting on $f: \mathbb{R}_{+} \rightarrow \mathbb{R}_{+}$, cf. $(K,+)$ in $\S 1$ :

$$
K(t, x) f:=\frac{f(x t)}{f(x)} .
$$

Suppose that $f$ is Karamata regularly varying, i.e. that, as $x \rightarrow \infty$,

$$
K(t, x) f:=\frac{f(x t)}{f(x)} \rightarrow K_{f}(t) . \quad(K, \times)
$$

Here we adopt a relatively new point of view on the classical theory, by making explicit use of what has so far been mostly implicit: the cocycle structure underlying the operator $K(t, x)$, cf. [Ell], [EllE], [Ost1]. It is this that characterizes the limit function $K_{f}$, the Karamata kernel of $f$. Indeed,

$$
\frac{f(x t s)}{f(x)}=\frac{f(x t s)}{f(x t)} \cdot \frac{f(x t)}{f(x)}: \quad K(s t, x)=K(s, x t) K(t, x),
$$

which in the limit yields the multiplicative Cauchy functional equation,

$$
K_{f}(s t)=K_{f}(s) K_{f}(t)
$$

We will need the Popa operation $\circ_{h}$ above to be associative, and (see Th. O below) this, as mentioned in $\S 2$ above, requires $h$ to satisfy the Gotab-Schinzel equation $(G S)$. Thus $(G S)$ expresses homomorphy in this context, which will occur after the passage to the limit $x \rightarrow \infty$. Before taking this limit, one has instead 'asymptotic associativity', or 'almost associativity'. The Popa notation $x \circ_{\varphi} t=x+t \varphi(x)$ describes a $t$-translation of $x$ modified locally at $x$, or 'accelerated at $x$ ' by reference to the 'accelerator' $\varphi$ (positive). We will need the 'rate of acceleration' and its asymptotic value for the $t$-translation:

$$
\eta_{x}(t), \text { or } \eta_{x}^{\varphi}(t),:=\frac{\varphi\left(x \circ_{\varphi} t\right)}{\varphi(x)}=\frac{\varphi(x+t \varphi(x))}{\varphi(x)} \rightarrow \eta(t), \text { or } \eta^{\varphi}(t)
$$

(assumed to exist), so that $\eta(t) \geq 0$. By the Uniform Convergence Theorem (UCT) [BinO7, §5], for $\varphi$ above Baire/measurable, convergence is necessarily locally uniform. The relevance of such convergence is witnessed by 
Theorem $\mathbf{O}$ [Ost3, Th. 0]. If $\varphi(x)=O(x)$ and $\eta_{x}(t) \rightarrow \eta(t)=\eta^{\varphi}(t)$, locally uniformly in $t$, then $\eta$ satisfies the Gotab-Schinzel functional equation

$$
\eta\left(s \circ_{\eta} t\right)=\eta(s) \eta(t) .
$$

As $\eta_{\infty}(t)=t$,

$$
\eta_{\infty}\left(s \circ_{\infty} t\right)=\eta_{\infty}(s) \eta_{\infty}(t)
$$

gives $\eta_{\infty}$ as an extreme case of $(G S)$.

Notational conventions. In Theorem $\mathrm{O}$ above $\eta_{x}$ contains the $x$ which tends to infinity. After this passage to the limit, below, attention focuses on the limit function $\eta(t)$ which will depend on a parameter $\rho$. We allow ourselves to denote this limit by $\eta_{\rho}(t)$, following $\S 2$, and let context speak for itself here.

For $\varphi$ Baire/measurable $\eta^{\varphi}$ is likewise Baire/measurable and so, as a solution of $(G S)$, continuous, by a theorem of Popa [Pop] and so $\eta^{\varphi} \in G S$. (Recall from $\S 2$ above that $G S:=\left\{\eta_{\rho}: \rho \in[0, \infty)\right\}$ denotes the family of continuous (positive) solutions of the equation $(G S)$.)

Below, we will encounter two auxiliary functions, $h$ and $\varphi$, the second of which will give such an $\eta$ asymptotically (so $\eta$ satisfies $(G S)$ and $\circ_{\eta}$ is associative).

For the purposes of combining an $s$ - and a $t$-translation, it is convenient to expand the accelerator notation to one parametrized locally at $x$ :

$$
s \circ_{\varphi x} t:=s+t \eta_{x}(s)=s+t \frac{\varphi(x+s \varphi(x))}{\varphi(x)} .
$$

So in the limit one has for $\eta=\eta^{\varphi}=\eta_{\rho}$ :

$$
\circ_{\varphi x} \rightarrow \circ_{\eta}=\circ_{\rho} .
$$

This justifies our earlier reference to 'asymptotic associativity'. A second reason for the term comes from a very convenient expression for a related form of associativity, one which otherwise the notation keeps hidden:

$$
\left(x \circ_{\varphi} b\right) \circ_{\varphi} a=x \circ_{\varphi}\left(b \circ_{\varphi x} a\right)
$$

(implicitly the justification is in the first line of the proof of the next result; cf. [BinO7, Prop. 2(ii)] for a proof). As an immediate application 
of this framework, we can rephrase the Beurling asymptotics, clarifying the underlying cocycle structure. These, as we will see, lead to specific functional equations, whose solutions are discussed in $\S 4$ below. See also the surveys [Brz1] and [Jab3]; cf. [Ost3].

Proposition 3 (Beurling regular variation). For the Beurling asymptotic operator $K^{\varphi}$ acting on $f: \mathbb{R}_{+} \rightarrow \mathbb{R}_{+}$,

$$
K^{\varphi}(t, x) f:=\frac{f(x+t \varphi(x))}{f(x)}=\frac{f\left(x \circ_{\varphi} t\right)}{f(x)},
$$

suppose that $f$ is Beurling regularly varying, i.e. that, as $x \rightarrow \infty$,

$$
K^{\varphi}(t, x) f:=\frac{f(x+t \varphi(x))}{f(x)}=\frac{f\left(x \circ_{\varphi} t\right)}{f(x)} \rightarrow K_{f}(t) .
$$

The corresponding cocycle structure is

$$
K^{\varphi}\left(t \circ_{\varphi x} s, x\right)=K^{\varphi}\left(s, x \circ_{\varphi} t\right) K^{\varphi}(t, x),
$$

leading in the limit to the Chudziak-Jabtonska equation

$$
K_{f}\left(t \circ_{\eta} s\right)=K_{f}(s) K_{f}(t)
$$

Proof. We have

$$
\frac{f(x+(s+t) \varphi(x))}{f(x)}=\frac{f\left(x+t \varphi(x)+\left(s / \eta_{x}(t)\right) \cdot \varphi(x+t \varphi(x))\right)}{f(x+t \varphi(x))} \cdot \frac{f(x+t \varphi(x))}{f(x)},
$$

so that

$$
K^{\varphi}(s+t, x)=K^{\varphi}\left(s / \eta_{x}(t), x+t \varphi(x)\right) K^{\varphi}(t, x) .
$$

Here replacing $s$ by $s \eta_{x}(t)$ yields

$$
K^{\varphi}\left(t+s \eta_{x}(t), x\right)=K^{\varphi}(s, x+t \varphi(x)) K^{\varphi}(t, x),
$$

as asserted. Taking limits yields $(C J)$.

We turn now to the general regular variation of the title and $\S 1$ (cf. [BinO14]). Following [Ost3], the auxiliary function $\varphi: \mathbb{R}_{+} \rightarrow \mathbb{R}_{+}$is selfequivarying, $\varphi \in S E$, if $\varphi(x)=O(x)$ and $\eta_{x}^{\varphi}(t) \rightarrow \eta(t)=\eta^{\varphi}(t)$, locally uniformly in $t$, as in Theorem $\mathrm{O}$. The auxiliary function $h$ will be Beurling 
regularly varying as in Prop. 1, i.e. $\varphi$-regularly varying, in the sense of [BinO5].

Proposition 4 (General regular variation). For the general asymptotics

$$
K_{h}^{\varphi}(t, x):=\frac{f(x+t \varphi(x))-f(x)}{h(x)} \rightarrow K_{f}(t),
$$

with $\varphi \in S E$, the corresponding cocycle structure is

$$
K_{h}^{\varphi}\left(t+s \eta_{x}(t), x\right)=K_{h}^{\varphi}\left(t \circ_{\varphi x} s, x\right)=K_{h}^{\varphi}(s, x) K^{\varphi}(t, x)+K_{h}^{\varphi}(t, x),
$$

leading in the limit to

$$
K_{f}(t+s \eta(t))=K_{f}(s) K_{h}(t)+K_{f}(t) \quad \text { with } \eta=\eta^{\varphi},
$$

or, equivalently, to the Beurling-Goldie equation satisfied by $K_{f}: \mathbb{G}_{\eta} \rightarrow \mathbb{G}_{\sigma}$ :

$$
K_{f}\left(t \circ_{\eta} s\right)=K_{f}(t) \circ_{\sigma} K_{f}(s), \quad \text { for } \sigma(z)=K_{h}\left(K_{f}^{-1}(z)\right) .
$$

Proof. Here the underlying cocycle structure mixes products with addition: with $y:=x \circ_{\varphi} t$,

$$
\begin{aligned}
K_{h}^{\varphi}(s+t, x) & =\frac{f(x+(s+t) \varphi(x))-f(x)}{h(x)} \\
& =\frac{f\left(x+t \varphi(x)+\left(s / \eta_{x}\right) \varphi\left(x \circ_{\varphi} t\right)\right)-f\left(x \circ_{\varphi} t\right)}{h\left(x \circ_{\varphi} t\right)} \cdot \frac{h\left(x \circ_{\varphi} t\right)}{h(x)}+\frac{f\left(x \circ_{\varphi} t\right)-f(x)}{h(x)} \\
& =\frac{f\left(y+\left(s / \eta_{x}\right) \varphi(y)\right)-f(y)}{h(y)} \cdot \frac{h\left(x \circ_{\varphi} t\right)}{h(x)}+K_{h}^{\varphi}(t, x) \\
& =K_{h}^{\varphi}\left(s / \eta_{x}, y\right) K^{\varphi}(t, x)+K_{h}^{\varphi}(t, x) .
\end{aligned}
$$

In the limit, since $x+t \varphi(x)=x(1+t \varphi(x) / x) \rightarrow \infty$ and $\varphi(x)=O(x)$,

$$
K_{f}(s+t)=K_{f}(s / \eta) K_{h}(t)+K_{f}(t),
$$

giving $(B G)$ as above.

Remarks. 1. In $(B G)$ above, $K_{h}$ and $K_{f}$ are positive (cf. Remark after Prop. 2) and so injective (by [Ost4, Th. 1]).

2. A measurable $\varphi: \mathbb{R}_{+} \rightarrow \mathbb{R}_{+}$is said to be Beurling slowly varying if, as with $S E$ above, $\eta^{\varphi}(t) \equiv 1$, and additionally $\varphi(x)=o(x)$ (that is, $\rho=0$ in the above). It is self-neglecting if the convergence $\eta_{x}(t) \rightarrow 1$ is locally uniformly in $t$; see [BinGT $\S 2.11$ ], [BinO5]. 


\section{Subadditivity in Popa groups}

Definition. For $\rho, \sigma \in[0, \infty]$, call $S: \mathbb{G}_{\rho} \rightarrow \mathbb{G}_{\sigma}$ subadditive (resp. additive) on a sub-semigroup $\Sigma$ of $\mathbb{G}_{\rho}$ if, for $x, y \in \Sigma$,

$$
S\left(x \circ_{\rho} y\right) \leq S(x) \circ_{\sigma} S(y) \quad \text { resp. } \quad S\left(x \circ_{\rho} y\right)=S(x) \circ_{\sigma} S(y),
$$

or in the notation of Theorem 2

$$
S\left(x+{ }_{\rho} y\right) \leq S(x)+{ }_{\sigma} S(y) \quad \text { resp. } \quad S\left(x+{ }_{\rho} y\right)=S(x)+{ }_{\sigma} S(y),
$$

As $\mathbb{G}_{0}=\mathbb{R}$ (the additive reals), when $\rho=\sigma=0$, this yields the usual notion of subadditivity, resp. additivity (on $\Sigma$ ).

Thus, $\|g\|_{\rho}$ is additive on the sub-semigroups $\mathbb{G}_{\rho}^{+}$.

In particular the solutions $K: \mathbb{G}_{\rho} \rightarrow \mathbb{G}_{\sigma}$ to the equation $(B G)$ are additive. For fixed $\rho \in[0, \infty]$ with $\sigma \in(0, \infty)$, their canonical form depends on a parameter $\kappa \in \mathbb{R}$ (Theorem 3 below, [Ost4], [Chu1,2]), as follows:

$$
\begin{aligned}
K_{\kappa}(t) & =\left\{\begin{array}{cc}
{\left[e^{\kappa t}-1\right] / \sigma,} & \text { if } \rho=0, \\
{\left[(1+\rho t)^{\kappa / \rho}-1\right] / \sigma,} & \text { if } \rho \in(0, \infty), \\
{\left[t^{\kappa}-1\right] / \sigma,} & \text { if } \rho=\infty,
\end{array}\right. \\
& =\eta_{\sigma}^{-1}\left(\eta_{\rho}(t)^{\kappa / \rho}\right), \text { for } \rho \in(0, \infty) .
\end{aligned}
$$

Above one has $\eta_{\rho}: \mathbb{G}_{\rho} \rightarrow(0, \infty)$, and $\eta_{\sigma}^{-1}: \mathbb{R}_{+} \rightarrow \mathbb{G}_{\sigma}$. The case $\kappa=0$ corresponds to the trivial solution $K \equiv 1_{\sigma}=0$.

In fact, for fixed $\rho, \sigma \in(0, \infty)$, the only additive functions bounded above are of this form, as below. Theorem 3, which follows, is our reformulation here of [Ost4, Prop. A]; cf. [Chu1,2].

In the context below of a homomorphism $\psi: \mathbb{G}_{\rho} \rightarrow \mathbb{G}_{\sigma}$, since $\eta_{\rho}$ and $\eta_{\sigma}$ both have range $\mathbb{R}_{+}$it is of significance that $\mathbb{R}_{+}$, viewed as the direct (inductive) limit of $\mathbb{G}_{\rho}$ (in the limit as $\rho \rightarrow \infty$ as in $\S 2$ ), may also be viewed as the limit of $\mathbb{G}_{\rho}$ under $\eta_{\rho}$ composed with scaling (of $\mathbb{R}_{+}$) by $1 / \rho$ as follows:

$$
\pi_{\rho}(x):=\eta_{\rho}(x) / \rho=x+(1 / \rho)>0 \text { for } x>\rho^{*} ;
$$

this is guided by the rescaling formula (resc) of $\S 2$. Here

$$
\pi_{\rho} \rightarrow \text { id as } \rho \rightarrow \infty
$$


Equally, in the limit as $\sigma \rightarrow \infty, \mathbb{R}_{+}$may also be viewed as the inverse limit of the groups $\mathbb{G}_{\sigma}$ under $\eta_{\sigma}^{-1}$ through a scaling (again of $\mathbb{R}_{+}$) in 'reverse', i.e. now by $\sigma$ :

$$
\eta_{\sigma}^{-1}(\sigma y)=(\sigma y-1) / \sigma=y-(1 / \sigma)>\sigma^{*} \text { for } y>0
$$

then

$$
\eta_{\sigma}^{-1}(\sigma y)=\pi_{-\sigma}: \quad \sigma(\cdot)=\eta_{\sigma} \pi_{-\sigma}(\cdot),
$$

i.e. a change of scale (multiplication by $\sigma$ in $\mathbb{R}_{+}$) factors through $\mathbb{G}_{\sigma}$.

When $\psi$ is factored, as below, through the linear mapping (of $\mathbb{R}$ into $\mathbb{R}$ ) $\Psi: x \mapsto \kappa x$, we can absorb the two scaling operations (in the domain and range of $\Psi)$ involving $\rho, \sigma \in(0, \infty)$ by rescaling $\kappa$ to $\kappa \sigma / \rho$.

Theorem 3 (Continuous Characterization). Take $\psi: \mathbb{G}_{\rho} \rightarrow \mathbb{G}_{\sigma}$ additive with $\rho, \sigma \in[0, \infty]$. Then the lifting $\Psi: \mathbb{R} \rightarrow \mathbb{R}$ of $\psi$ to $\mathbb{R}$ defined by the canonical isomorphisms $\log$, exp, $\left\{\eta_{\rho}: \rho \in(0, \infty)\right\}$ is bounded above on $\mathbb{G}_{\rho}$ iff $\Psi$ is bounded above on $\mathbb{R}$, in which case $\Psi$ and $\psi$ are continuous. Then for some $\kappa \in \mathbb{R}$ one has the characterization tabled below, which varies continuously in the parameters.

\begin{tabular}{|l|l|l|l|}
\hline Popa parameter & $\sigma=0$ & $\sigma \in(0, \infty)$ & $\sigma=\infty$ \\
\hline$\rho=0$ & $\kappa t$ & $\eta_{\sigma}^{-1}\left(e^{\kappa \sigma t}\right)$ & $e^{\kappa t}$ \\
\hline$\rho \in(0, \infty)$ & $\log \eta_{\rho}(t)^{\kappa / \rho}$ & $\eta_{\sigma}^{-1}\left(\eta_{\rho}(t)^{\kappa \sigma / \rho}\right)$ & $\eta_{\rho}(t)^{\kappa / \rho}$ \\
\hline$\rho=\infty$ & $\log t^{\kappa}$ & $\eta_{\sigma}^{-1}\left(t^{\kappa \sigma}\right)$ & $t^{\kappa}$ \\
\hline
\end{tabular}

Proof. We begin with the tabled forms. The canonical isomorphisms are order-preserving and continuous. For $\rho, \sigma \in(0, \infty)$ the lifting is given by

$$
\Psi(\cdot)=\log \eta_{\sigma} \psi \eta_{\rho}^{-1} \exp (\cdot)
$$

and this still holds for extreme values of $\rho, \sigma$ with exp, log replacing $\eta_{0}, \eta_{\infty}$. For $\Psi(x)=(\kappa \sigma / \rho) x$ (or $\Psi(x)=\kappa x$, as the case may be), a routine calculation confirms $\psi$ to be as in the table:

$$
\psi(y)=\eta_{\sigma}^{-1} \exp (\kappa \sigma / \rho) \log \eta_{\rho}(y)=\eta_{\sigma}^{-1}\left(\eta_{\rho}(y)^{\kappa \sigma / \rho}\right) \in \mathbb{G}_{\sigma} .
$$

Next we consider passages to the various limits. 
Direct passage from the middle to the first column, and likewise from the middle to the first row, follows from L'Hospital's Rule (see the arXiv version). No scaling of $\kappa$ is needed in the passage to the third row, where in the limit for $\rho \rightarrow \infty$, proceeding as with the norm (in Prop. 1(c)),

$$
\eta_{\rho}\left(t \circ 1_{\rho}^{-1}\right)^{\kappa}=\left[\eta_{\rho}(t) / \eta_{\rho}\left(1_{\rho}\right)\right]^{\kappa}=[(1+\rho t) /(1+\rho)]^{\kappa} \rightarrow t^{\kappa} .
$$

Passage from the middle to the third columns recognizes that the passage from $\mathbb{G}_{\sigma}$ to $\mathbb{R}_{+}$requires rescaling (as noted above):

$$
\begin{aligned}
\lim _{\sigma \rightarrow \infty} \eta_{\sigma}^{-1}\left(\sigma \eta_{\rho}(t)^{\kappa / \rho}\right) & =\lim _{\sigma \rightarrow \infty}\left[\eta_{\rho}(t)^{\kappa / \rho}-1 / \sigma\right]=\eta_{\rho}(t)^{\kappa / \rho} \text { for } \rho \in(0, \infty) ; \\
\lim _{\sigma \rightarrow \infty} \eta_{\sigma}^{-1}\left(\sigma t^{\kappa}\right) & =\lim _{\sigma \rightarrow \infty}\left[t^{\kappa}-1 / \sigma\right]=t^{\kappa} \text { for } \rho=\infty .
\end{aligned}
$$

The remaining case $\rho=0$ requires also a conjugate action in $\mathbb{G}_{\rho}$; take $\rho \rightarrow 0+$ and $\sigma \rightarrow \infty$; then $\sigma \kappa / \rho \rightarrow \infty$, and so

$$
\lim _{\substack{\rho \rightarrow 0+\\ \sigma \rightarrow \infty}} \eta_{\sigma}^{-1}\left(\sigma \eta_{\rho}(t / \sigma)^{\kappa \sigma / \rho}\right)=\lim _{\substack{\rho \rightarrow 0+\\ \sigma \rightarrow \infty}}\left[(1+\kappa t /(\kappa \sigma / \rho))^{\kappa \sigma / \rho}-1 / \sigma\right]=e^{\kappa t} .
$$

Remarks. 1. Direct passage between the first and the third columns is effected via $\exp / \log$.

2. The case $\rho=0$ and $\sigma=\infty$ (the top right-hand entry above) gives $\psi: \mathbb{R} \rightarrow \mathbb{R}_{+}$of the form $\psi(t)=e^{\kappa t}$. This corresponds to Beurling $\varphi$-RV for $\varphi \in S N$ : specializing $(G)$ to $h=1$ and rewriting multiplicatively gives

$$
f(x+t \varphi(x)) / f(x) \rightarrow e^{\kappa t} .
$$

See de Haan [dHa], [BinGT, $\S 3.10]$, [BinO5]. For applications to the limit theory of record values in probability see [LanS]; cf. [Arr], [BarAE], [FalKCP1,2].

Definition. Call $S: \mathbb{G}_{\rho} \rightarrow \mathbb{G}_{\sigma}$ additively bounded on $\Sigma$ if for some $\kappa \in \mathbb{R}$

$$
S(s) \leq K_{\kappa}(s) \quad(s \in \Sigma) .
$$

This lifts to the Popa context the notion of linear boundedness used in [BinO10].

The four results below are liftings of results known on $\mathbb{R}$ to a Popa-group setting ('P for Popa'); they correspond respectively to Prop. 5, Theorem 0, 
Theorem $0^{\prime}$; and Theorem $0^{+}$of [BinO10]. Recall that $B_{\delta}(x)$ is the open interval (ball) about $x$ of radius $\delta$ in the relevant group norm $\|\cdot\|_{\rho}$.

Theorem PR. For $\rho, \sigma \in[0, \infty]$ and $S: \mathbb{G}_{\rho} \rightarrow \mathbb{G}_{\sigma}$ subadditive:

(i) if $S$ is bounded above on some interval, say by $M>0$ on $B_{\delta}(a)$, then for any $b \in \mathbb{G}_{\rho}$

$$
S(b \circ a) \circ_{\sigma} M_{\sigma}^{-1} \leq S(x) \leq S\left(b \circ a_{\rho}^{-1}\right) \circ_{\sigma} M \quad\left(x \in B_{\delta}(b)\right)
$$

(with $M_{\sigma}^{-1}$ etc. the inverses in the corresponding groups); in particular it is locally bounded.

(ii) If $S$ is locally bounded, then $\liminf _{t \rightarrow 1_{\rho}} S(t) \geq 1_{\sigma}$, so $S\left(1_{\rho}+\right)=1_{\sigma}$ if $S$ satisfies the Heiberg-Seneta condition

$$
\lim \sup _{x \downarrow 1_{\rho}} S(x) \leq 1_{\sigma} .
$$

Proof. Since the Popa groups are order isomorphic, w.l.o.g. we may assume $\rho, \sigma \in(0, \infty)$. This is then a lifting of [BinO10, Prop. 5].

For a definition of the local $S W$ property below and the notation $\Sigma_{z}^{\delta}=$ $\Sigma \cap B_{\delta}(z)$, see after Theorem 5 in $\S 5$.

Theorem P1. For $\rho, \sigma \in[0, \infty]$ and $S: \mathbb{G}_{\rho} \rightarrow \mathbb{G}_{\sigma} \cup\left\{\sigma^{*},+\infty\right\}$ subadditive on $\mathbb{G}_{\rho}$ with $S\left(1_{\rho}+\right)=S\left(1_{\rho}\right)=1_{\sigma}: S$ is continuous at $1_{\rho}$ iff $S\left(z_{n}\right) \rightarrow 1_{\sigma}$, for some sequence $z_{n} \uparrow 1_{\rho}$, and then $S$ is continuous everywhere, if finite-valued.

Proof of Theorem P1. As before, w.l.o.g. we may assume $\rho, \sigma \in(0, \infty)$. This is now as in [BinO10, Th. 0], mutatis mutandis, as the group order is the usual order on the line (Prop. 2), and with $-x$ etc. replaced by $x_{\rho}^{-1}$ (equivalently by $-{ }_{\rho} x$, cf. Theorem 2 ).

Theorem P2 [BinO8, Th. 3]. For $\rho, \sigma \in[0, \infty]$, if $S: \mathbb{G}_{\rho} \rightarrow \mathbb{G}_{\sigma}$ is subadditive on $\mathbb{G}_{\rho}$ with $S\left(1_{\rho}\right)=1_{\sigma}$ and there is a symmetric set $\Sigma$ containing $1_{\rho}$ such that:

(i) $S$ is continuous at $1_{\rho}$ on $\Sigma$;

(ii) for all small enough $\delta>0, \Sigma_{1_{\rho}}^{\delta}$ is locally Steinhaus-Weil

- then $S$ is continuous at $1_{\rho}$ and so everywhere.

In particular, this conclusion holds if there is a symmetric set $\Sigma$, Baire/measurable and non-negligible in each $\left(1_{\rho}, \delta\right)$ for $\delta>1_{\rho}$, on which

$S(u)=K_{\kappa_{ \pm}}(u)$ for some $\kappa_{ \pm} \in \mathbb{R}$ and all $u \in \mathbb{G}_{\rho}^{+} \cap \Sigma$, or all $u \in \mathbb{G}_{\rho}^{-} \cap \Sigma$ resp. 
Proof of Theorem P2. W.l.o.g. $\sigma \in(0, \infty)$, as the case $\sigma=0$ is similar but simpler, and on using exp the latter implies also the case $\sigma=\infty$. Using a transformation of the domain uder log or exp may also reduce the theorem to the case $\rho \in(0, \infty)$ for which $1_{\rho}=0$. Since $S \mid \Sigma$ is continuous at 0 it is bounded above on $\Sigma_{\delta}:=\Sigma \cap\left(\delta_{\rho}^{-1}, \delta\right),=\left(\Sigma_{\delta}\right)_{\rho}^{-1}$, for some $\delta>0$; but $\Sigma_{\delta} \circ \Sigma_{\delta}$ contains an interval, so $S$ is bounded on an interval, and so locally bounded by Theorem PR(i). If $S$ is not continuous at $1_{\rho}$, then by Theorem $\operatorname{PR}\left(\right.$ ii) $\lambda_{+}:=\limsup _{t \rightarrow 0} S(t)>\liminf _{t \rightarrow 0} S(t) \geq 0$, so $\lambda_{+}>0$. Choose a null sequence $\left\{z_{n}\right\}$ with $S\left(z_{n}\right) \rightarrow \lambda_{+}>0$. Let $\varepsilon:=\min \left\{\lambda_{+} / 6,1 / \sigma\right\}$. W.l.o.g. $S\left(z_{n}\right)>\lambda_{+}-\varepsilon$ for all $n$. By continuity on $\Sigma$ at 0 , there is $\delta>0$ with $|S(t)|<\varepsilon$ for $t \in \Sigma_{\delta}$. As before and using symmetry, $\Sigma_{\delta} \circ \Sigma_{\delta}=\Sigma_{\delta} \circ\left(\Sigma_{\delta}\right)_{\sigma}^{-1}$ contains an interval $I$ around 0 . For any $n$ with $z_{n} \in I$, there are $u_{n}, v_{n} \in \Sigma_{\delta}$ with $z_{n}=u_{n} \circ_{\rho} v_{n}$; then, as $\varepsilon \leq 1 / \sigma$,

$$
\begin{aligned}
S\left(z_{n}\right) & \leq S\left(u_{n}\right) \circ_{\sigma} S\left(v_{n}\right)=S\left(u_{n}\right)+S\left(v_{n}\right)\left(1+\sigma S\left(u_{n}\right)\right) \\
& \leq \varepsilon(2+\sigma \varepsilon) \leq 3 \varepsilon \leq \lambda_{+} / 2 .
\end{aligned}
$$

So

$$
5 \lambda_{+} / 6 \leq \lambda_{+}-\varepsilon<S\left(z_{n}\right) \leq S\left(u_{n}\right) \circ_{\sigma} S\left(v_{n}\right) \leq 3 \varepsilon \leq \lambda_{+} / 2,
$$

a contradiction as $\lambda_{+}>0$. So $S$ is continuous at 0 and so continuous everywhere (as in Theorem P1):

$$
{ }_{-} S\left(-{ }_{\rho} h\right) \leq S\left(x+{ }_{\rho} h\right)-{ }_{\sigma} S(x) \leq S(h) .
$$

The last part follows since $\Sigma \cap(0, \delta)$, being Baire/measurable and nonnegligible, has the SW property for each $\delta>0$.

Theorem P3. For $\rho, \sigma \in[0, \infty]$, let $\Sigma \subseteq\left[1_{\rho}, \infty\right)$ be locally $S W$ accumulating at $1_{\rho}$. Suppose $S: \mathbb{G}_{\rho} \rightarrow \mathbb{G}_{\sigma}$ is subadditive on $\mathbb{G}_{\rho}$ with $S\left(1_{\rho}\right)=1_{\sigma}$ and $S \mid \Sigma$ is additively bounded above by $G(x):=K_{\kappa}(x)$, i.e. $S(s) \leq K_{\kappa}(s)$ for some $\kappa$ and all $s \in \Sigma$, so that in particular,

$$
\lim \sup _{s \downarrow 0, s \in \Sigma} S(s) \leq 1_{\sigma} .
$$

Then $S(x) \leq K_{\kappa}(x)$ for all $x>1_{\rho}$, so

$$
\lim \sup _{x \downarrow 1_{\rho}} S(x) \leq 1_{\sigma},
$$

and so $S\left(1_{\rho}+\right)=1_{\sigma}$. 
In particular, if furthermore there exists a sequence $\left\{z_{n}\right\}_{n \in \mathbb{N}}$ with $z_{n} \uparrow 1_{\rho}$ and $S\left(z_{n}\right) \rightarrow 1_{\sigma}$, then $S$ is continuous at $1_{\rho}$ and so everywhere.

Proof of Theorem P3. As before, w.l.o.g. we may assume $\rho, \sigma \in(0, \infty)$. We are to show that $S(t) \leq K_{\kappa}(t)$ for all $t$. We may begin with the simplifying assumption that $K \equiv 1_{\sigma}=0$, i.e. that $\kappa=0$, since $\bar{S}(t):=S(t) \circ_{\sigma}\left(K_{\kappa}(t)\right)_{\sigma}^{-1}$ is linearly bounded above by $1_{\sigma}=0$ on $\Sigma$, and $\bar{S}$ is subadditive, as $K$ is additive. Then for $u, v \in \mathbb{G}_{\rho}$

$$
\bar{S}(u \circ v):=S(u \circ v) \circ_{\sigma} K_{\kappa}\left(u \circ_{\rho} v\right)_{\sigma}^{-1} \leq S(u) \circ_{\sigma} S(v) \circ_{\sigma} K(u)_{\sigma}^{-1} \circ_{\sigma} K(v)_{\sigma}^{-1} .
$$

From now on the proof follows that of $\left[\mathrm{BinO} 10\right.$, Th. $\left.0^{+}\right]$, mutatis mutandis (interpreting + as $+_{\rho}$ and - as $-\rho$ as in Theorem 2).

\section{Functional inequalities from asymptotic ac- tions: the Goldie argument}

We return to the Karamata asymptotic operator $K$ acting on $f: \mathbb{R}_{+} \rightarrow \mathbb{R}_{+}$, as in $(K, \times)$ of $\S 3$, but we now apply a natural alternative to the limits of $\S 3$ when they cannot be assumed to exist. This is provided by the lim sup operation, which in the Karamata setting is given by

$$
K^{*}(t) f:=\lim \sup K(t, x) f=\limsup \frac{f(x t)}{f(x)}:=K_{f}^{*}(t) .
$$

This leads to an operator domain defined by

$$
\mathbb{A}_{f}:=\left\{u: K_{f}(u):=\lim f(x u) / f(x) \text { exists and is finite }\right\} .
$$

This is a subgroup of $\mathbb{R}_{+}$. For positive functions $f$, one has

$$
\begin{gathered}
\limsup \frac{f(x s t)}{f(x)} \leq \limsup \frac{f(x t s)}{f(x t)} \cdot \limsup \frac{f(x t)}{f(x)}: \\
K(s t, x) \leq K(s, x t) K(t, x), \quad K^{*}(s t) f \leq K^{*}(s) f \cdot K^{*}(t) f .
\end{gathered}
$$

Here the limsup yields the multiplicative Cauchy functional inequality [Kuc, Ch. 16],

$$
K_{f}^{*}(s t) \leq K_{f}^{*}(s) K_{f}^{*}(t)
$$


as well as a pair of equations restricted to $\mathbb{A}_{f}$ :

$$
\left.\begin{array}{ccc}
K_{f}(s t) & = & K_{f}(s) K_{f}(t), \\
K_{f}(t) & = & K_{f}^{*}(t)
\end{array}\right\} \quad\left(s, t \in \mathbb{A}_{f}\right) .
$$

One seeks side-conditions on $f$ and imposes a density condition on $\mathbb{A}_{f}$ to deduce that $\mathbb{A}_{f}=\mathbb{R}_{+}$.

For the general asymptotics, with $\varphi \in S E$,

$$
K_{h \varphi}^{*}(t) f:=\lim \sup \frac{f(x+t \varphi(x))-f(x)}{h(x)},
$$

there is a corresponding operator domain defined (omitting $\varphi$ when clear from context) by

$$
\mathbb{A}_{h f}:=\left\{u: K_{h f}(u):=\lim [f(x+u \varphi(x))-f(x)] / h(x) \text { exists and is finite }\right\} .
$$

Writing

$$
K_{h f}^{*}(t):=K_{h \varphi}^{*}(t) f,
$$

as in $(C F I)$ above, there is also a functional inequality:

$$
K_{h f}^{*}(t+s \eta(t)) \leq K_{h f}^{*}(s) K_{h}(t)+K_{h f}^{*}(t), \quad \text { with } K_{h}(t):=\lim h\left(x \circ_{\varphi} t\right) / h(x),
$$

where $\eta=\eta^{\varphi}$ and $K_{h}$ is assumed to exist for all $t$ (as in Prop. 4). The inequality may be reformulated in Popa-group language as the BeurlingGoldie inequality satisfied by $K_{f}^{*}: \mathbb{G}_{\eta} \rightarrow \mathbb{G}_{\sigma}:$

$$
K_{h f}^{*}\left(t \circ_{\eta} s\right) \leq K_{h f}^{*}(t) \circ_{\sigma} K_{h f}^{*}(s), \quad \text { for } \sigma(z)=K_{h}\left(K_{h f}^{*-1}(z)\right) .
$$

However, there is no immediate justification for $\mathbb{A}_{h f}$ being a subgroup, short of further hypotheses. Either an imposition of good behaviour of the limit, such as local uniformity in $u \in \mathbb{A}_{h f}$, is needed, thus narrowing the domain, or a presumption of topologically good character of the domain itself, such as requiring $\mathbb{A}_{h f}$ to contain a non-meagre Baire subset. The latter may draw on additional axioms of set theory, for which see [BinO12]. For an extensive study of such good behaviour and character, see [BinO7].

Henceforth we take for granted a domain $\mathbb{A}$ that is a dense subgroup of an appropriate Popa group $\mathbb{G}$, and a side-condition of right-sided continuity at $1_{\mathbb{G}}$ imposed on $K_{h f}^{*}$ (so on $\left.\mathbb{R}_{+}\right)$: see the various conditions $(H S)$ in Th. PR above and in Th. 5 and the related $(S W-H S)$ in Th. 6 below. 
Above we had the Beurling-Goldie equation $(B G)$. Below, we restrict one or both of the arguments $u$ and $v$ to $\mathbb{A}$, obtaining the 'singly conditioned' and 'doubly conditioned' Beurling-Goldie equations: see $\left(B G_{\mathbb{A}}\right)$ and the deduction $\left(B G_{\mathbb{G G}}\right)$. For the origins of the Goldie argument, see the Remark after Theorem 4 below.

Remark. The following inequality, appropriate to the case $\rho=0$ of $K_{-\gamma}$, shows why the subadditivity of $\left(1-e^{-\gamma u}\right) / \gamma$ is restricted to the domain $\mathbb{R}_{+}$ for $\gamma>0$ and clarifies the Lemma below:

$$
0<\left(1-e^{-\gamma u}\right)\left(1-e^{-\gamma u}\right)=1-e^{-\gamma u}-e^{-\gamma v}+e^{-\gamma(u+v)} .
$$

Lemma (cf. [HilP, Th. 7.2.5, p. 239]). A differentiable function $f$ concave on $[0, \infty)$ with $f(0) \geq 0$ is subadditive on $[0, \infty)$. However, if $f$ with $f(0)=0$ is strictly concave on $(-\delta, \infty)$ for some $\delta>0$, then $f$ is not subadditive on $(-\delta, \infty)$.

In particular, for $\gamma \neq 0$ and $\rho \in(0, \infty), K_{-\gamma}(t):=\left[1-(1+\rho t)^{-\gamma / \rho}\right] / \gamma$ is subadditive on $[0, \infty)$ for $\gamma \geq-\rho$, but not subadditive on $\mathbb{G}_{\rho}$ for $\gamma>-\rho$; likewise for $\left(1-e^{-\gamma t}\right) / \gamma$ for $\gamma>0$.

Proof. Fix $v>0$; then $F(u):=f(u+v)-f(u)-f(v)$ is decreasing for $u \geq 0$, as $f^{\prime}(x)$ is decreasing and

$$
F^{\prime}(u)=f^{\prime}(u+v)-f^{\prime}(u) \leq 0 .
$$

But $F(0)=-f(0) \leq 0$, so $F(u) \leq F(0) \leq 0$ for $u>0$ and so $f$ is subadditive on $[0, \infty)$.

Now suppose further that $f(0)=0$ and that $f$ is strictly concave on $I:=(-\delta, \infty)$, so that $f^{\prime}(x)$ is strictly decreasing also on $I$. Again we fix $v$, but take $\delta<v<0$. Then for any $u \geq 0$, one has $F^{\prime}(u)>0($ as $u+v<u)$, and so $F(u)>0$. Then $f(u+v)>f(u)+f(v)$, so $f$ is not subadditive on $I$.

The first special case of subadditivity on $[0, \infty)$ follows, since $K_{-\gamma}(0)=0$ and $K_{-\gamma}^{\prime \prime}(t):=-(\rho+\gamma)(1+\rho t)^{-2-\gamma / \rho} \leq 0$ for all $t \in \mathbb{G}_{\rho}$ for $\gamma \geq-\rho$. However, for $\gamma+\rho>0, K_{-\gamma}(t)$ is strictly concave on $\mathbb{G}_{\rho}$ and so not subadditive on $\mathbb{G}_{\rho}$. $\left[K_{-\gamma}(t)=t\right.$, for $-\gamma / \rho=1$.]

Remark. The case $-\rho \leq \gamma<0$, which can arise in the context of the Lemma, cannot arise in the context of Theorem 4 below. The analysis there focuses on $\mathbb{G}_{\rho}^{+}$as defined in $\S 2$ (since conventionally, the main concern in 
regular variation is behaviour 'far enough to the right' on $\mathbb{R}$ ); the results, however, extend to the whole of $\mathbb{G}_{\rho}$ by exploiting 'pseudo-symmetry' (below).

We note that the result below naturally extends to any locally compact abelian group $(\mathbb{G}, \circ)$ embeddable in $\mathbb{R}$ for which the group action has relative invariance (see the remarks preceding and comment following Theorem 2).

Theorem 4 (Generalized Goldie Theorem, cf. [BinO6, Th. 3]). If for $\rho \in[0, \infty]$ and $\mathbb{A}$ a dense subgroup of $\mathbb{G}_{\rho}$ :

(i)(a) $F^{*}: \mathbb{G}_{\rho} \rightarrow \mathbb{G}_{0}=\mathbb{R}$ is subadditive, meaning $F^{*}\left(x \circ_{\rho} y\right) \leq F^{*}(x)+F^{*}(y)$ for $x, y \in \mathbb{G}_{\rho}$;

(b) $F^{*}$ is positive on $\mathbb{G}_{\rho}^{+}$with $F^{*}\left(1_{\rho}+\right)=0$;

(ii) $F^{*}$ satisfies the singly-conditioned Beurling-Goldie equation

$$
F^{*}\left(u \circ_{\rho} v\right)=g(v) K(u)+F^{*}(v) \quad(u \in \mathbb{A})\left(1_{\rho}<_{\rho} v\right) \quad\left(B G_{\mathbb{A}}\right)
$$

for some non-zero $K$, with $g$ a continuous function on $\mathbb{G}_{\rho}^{+} \cup\left\{1_{\rho}\right\}$ taking the value 1 at $1_{\rho}$ only;

(iii) $F^{*}$ extends $K$ on $\mathbb{A}$ :

$$
F^{*}(x)=K(x) \quad(x \in \mathbb{A})
$$

(so in particular $\left(B G_{\mathbb{A}}\right)$ may be viewed as a functional equation defining $F^{*}$ );

- then for some $c>0, \gamma>0$,

(a) $g$ satisfies $g\left|\mathbb{G}_{\rho}^{+}=g_{-\gamma}\right| \mathbb{G}_{\rho}^{+}$, where

$$
g_{-\gamma}(x):=\left\{\begin{array}{cc}
e^{-\gamma x}, & \text { if } \rho=0 \\
(1+\rho x)^{-\gamma / \rho}, & \text { if } \rho>0 \\
x^{-\gamma}, & \text { if } \rho=\infty
\end{array}\right.
$$

(b) $F^{*}(u):=c[g(u)-1] / \gamma$ for $1_{\rho} \leq_{\rho} u$, and so, for all $x \in \mathbb{G}_{\rho}$,

$$
F^{*}(x)=c K_{-\gamma}(x):=\left\{\begin{array}{cc}
c\left(1-e^{-\gamma x}\right) / \gamma, & \text { if } \rho=0 \\
c\left[1-(1+\rho x)^{-\gamma / \rho}\right] / \gamma, & \text { if } \rho>0 \\
c\left(1-x^{-\gamma}\right) / \gamma, & \text { if } \rho=\infty
\end{array}\right.
$$

That is,

$$
F^{*}\left(u \circ_{\rho} v\right)=g_{-\gamma}(v) F^{*}(u)+F^{*}(v) \quad\left(u, v \in \mathbb{G}_{\rho}\right) . \quad\left(B G_{\mathbb{G} \mathbb{G}}\right)
$$


Proof. Notice that $\left(B G_{\mathbb{A}}\right)$ gives information about $g$ only on $\mathbb{R}_{+}$or $[1, \infty)$ as the case may be.

We write $\mathbb{G}$ for $\mathbb{G}_{\rho}$ and $\mathbb{G}_{+}$for $\mathbb{G}_{\rho}^{+}$etc. (so $\mathbb{G}_{-}=\mathbb{G} \backslash\left(\mathbb{G}_{+} \cup\left\{1_{\rho}\right\}\right.$ ) and $\eta$ denotes $\eta_{\rho}$ ). We may write $+_{\rho}$ for $\circ_{\rho}$, as in Theorem 2 , or even + if no misunderstanding arises (so that - may stand for $-\rho$ ); the symbol $\sum$ is only used for ordinary addition on $\mathbb{R}$. When the group context allows, we rely on the context to identify whether 0 denotes the additive or multiplicative neutral element. Below, for $1_{\rho}<_{\rho} \delta$ and $n=0,1,2, \ldots, n \delta$ will mean the $n$-fold sum in $\mathbb{G}$ (inductively defined so that $0 \delta=1_{\rho}$ and $n \delta=(n-1) \delta \circ_{\rho} \delta$ ).

We proceed in three steps as follows.

Step 1 . We begin by proving that the restriction $F_{\mathbb{A}}^{*}:=F^{*} \mid \mathbb{A}$ is pseudosymmetric:

$$
F^{*}(-u)=-F^{*}(u) / g(u) \quad\left(u \in \mathbb{A} \cap \mathbb{G}_{+}\right) .
$$

From here we deduce that $F_{\mathbb{A}}^{*}$ is continuous.

Step 2. We prove the form that $F^{*}$ takes, first (a) on $\mathbb{A}_{+}:=\mathbb{A} \cap \mathbb{G}_{+}$and then (b) on $\mathbb{G}_{+}$.

Step 3. We use the results in Step 2 and pseudo-symmetry to find that $F^{*}$ takes the same form as in Step 2, first (a) on $\mathbb{A}_{-}:=\mathbb{A} \cap \mathbb{G}_{-}$and finally (b) on $\mathbb{G}_{-}$. Thus the same form holds on all of $\mathbb{G}$.

Step 1 . We begin by noting that $F^{*}(0)=0$; indeed, as $F^{*}(0)=K(0)$ by (iii),

$$
0=F^{*}(0+)=\lim _{v \downarrow 0} F^{*}(0+v)=g(0) F^{*}(0)+F^{*}(0+)=F^{*}(0) .
$$

So, as $F_{\mathbb{A}}^{*}=K \mid \mathbb{A}$, for $u \in \mathbb{A}_{+}$,

$$
0=F^{*}(0)=F^{*}(-u+u)=g(u) F^{*}(-u)+F^{*}(u),
$$

yielding pseudo-symmetry of $F_{\mathbb{A}}^{*}$. So $F_{\mathbb{A}}^{*}$ is continuous at 0 , as

$$
F_{\mathbb{A}}^{*}(0-)=\lim _{v \downarrow 0 \& v \in \mathbb{A}} F^{*}(-v)=\lim _{v \downarrow 0 \& v \in \mathbb{A}}-F^{*}(v) / g(v)=-F^{*}(0+)=0 .
$$

Next, $F^{*}$ is right-continuous at all points of $\mathbb{A}$ (so in particular $F_{\mathbb{A}}^{*}$ is rightcontinuous), since for any $u \in \mathbb{A}$

$$
F^{*}(u+)=\lim _{v \downarrow 0} F^{*}(u+v)=g(0) F^{*}(u)+F^{*}(0+)=F^{*}(u) .
$$


Furthermore, the right-sided restriction $F^{*} \mid \mathbb{A}_{+}$is left-continuous: for $u \in \mathbb{A}_{+}$ by right-continuity of $F_{\mathbb{A}}^{*}$ (using $v<u$ )

$$
\begin{aligned}
-F^{*}(u) / g(u) & =F^{*}(-u)=F_{\mathbb{A}}^{*}(-u+)=\lim _{v \downarrow 0 \& v \in \mathbb{A}} F^{*}(-u+v)=-\lim _{v \downarrow 0 \& v \in \mathbb{A}}\left[F^{*}(u-v) / g(u-v)\right] \\
& =-\left[\lim _{v \downarrow 0 \& v \in \mathbb{A}} F^{*}(u-v)\right] / g(u)=-F_{\mathbb{A}}^{*}(u-) / g(u) .
\end{aligned}
$$

Combining, $F^{*} \mid \mathbb{A}_{+}$is continuous, since for $u \in \mathbb{A} F^{*}(u+)=F^{*}(u)=$ $\lim _{v \downarrow 0 \& v \in \mathbb{A}} F^{*}(u-v)$. We transfer this to $\mathbb{A}_{-}$by noting that for $u \in \mathbb{A}_{+}$

$F_{\mathbb{A}}^{*}(-u \pm)=\lim _{v \downarrow 0 \& v \in \mathbb{A}} F^{*}(-u \pm v)=-\lim _{v \downarrow 0 \& v \in \mathbb{A}}\left[F^{*}(u \mp v) / g(u \mp v)\right]=-F^{*}(u) / g(u)=F_{\mathbb{A}}^{*}(-u)$.

Combining all the continuity results, $F_{\mathbb{A}}^{*}$ is continuous.

Step 2. We claim first that

$$
0<g(u)<1 \quad\left(u \in \mathbb{A}_{+}\right) .
$$

To prove the right-hand side, recall from hypothesis (iii) that $F^{*}|\mathbb{A}=K| \mathbb{A}$; so for $u, v \in \mathbb{A}_{+}$, by $\left(B G_{\mathbb{A}}\right)$ and subadditivity on $\mathbb{G}_{+}$,

$$
g(u) K(v)+K(u)=K\left(v \circ_{\eta} u\right) \leq K(v)+K(u) ;
$$

as $g$ never takes the value 1 except at 0 , the claim now follows since $K(v)>0$.

Actually, as $g$ is continuous and $\mathbb{A}$ is dense, this inequality extends to $\mathbb{G}_{+}$.

To prove the left-hand inequality, note that for $u, v \in \mathbb{A}_{+}$,

$$
g(u) K(v)+K(u)=K(u+v)=g(v) K(u)+K(v) .
$$

So, as $g<1$ on $\mathbb{A}_{+}$and $K \mid \mathbb{A}_{+}$is positive,

$$
\frac{K(u)}{1-g(u)}=\frac{K(v)}{1-g(v)}=\text { const. }=\kappa,
$$

say, for some $\kappa>0$. So

$$
K(u)=\kappa[g(u)-1] \quad\left(u \in \mathbb{A}_{+}\right) .
$$

Substitution into $B G_{\mathbb{A}}$ yields for $u, v \in \mathbb{A}_{+}$

$$
\kappa[g(u+v)-1]=g(v) \kappa[g(u)-1]+\kappa[g(v)-1]: \quad g(u+v)=g(u) g(v) .
$$


As $g(0)=1$, by continuity, $0<g(v)$ for $v \in(0, \delta)$ for some $\delta>0$. We show this implies positivity on $\mathbb{A}_{+}$. For $\delta \leq u \in \mathbb{A}_{+}$and any $v \in(0, \delta) \cap \mathbb{A}$, noting that $1<\delta / v$ and $1<u / v$, choose $n \in \mathbb{N}$ with $u / v \leq n<(u+\delta) / v$. Then

$$
0<n v-u<\delta
$$

so, since $n v-u \in(0, \delta) \cap \mathbb{A}$,

$$
0<g(v)^{n} / g(n v-u)=g(n v) / g(n v-u)=g(u) .
$$

Step 2(a). Now we work in $\mathbb{A}_{+}$, using integrals defined on $\mathbb{G}_{+}$. Recalling that 0 here and in the integral below denotes $1_{\rho}$, for $x>0$, put

$$
G(x):=\int_{0}^{x} g(t) \mathrm{d} t / \eta(t): \quad G^{\prime}(x)=g(x) / \eta(x) .
$$

We now mimic the Goldie proof of [BinGT, §3.2.1] (extending [BinO6, Th. 3] to the current Popa context). Here we use the density of $\mathbb{A}$ and the agreement of $\leq_{\rho}$ and $\leq$ (Prop. 2). Fix $u_{0} \in \mathbb{A}_{+}$and take $u \in \mathbb{A}_{+}$. For $\delta>0$, define $i=i(\delta) \in \mathbb{N}$ for $\delta>0$ so that $(i-1) \delta \leq u<i \delta$. Likewise for $u_{0}$ define $j=i_{0}(\delta) \in \mathbb{N}$, i.e. so that $(j-1) \delta \leq u_{0}<j \delta$. As $g$ takes the value 1 at $1_{\rho}$ only, put $c_{0}:=K\left(u_{0}\right) /\left[g\left(u_{0}\right)-1\right]$. For $\delta \in \mathbb{A}_{+}$and $m \in \mathbb{N}$

$$
F^{*}(m \delta)-F^{*}((m-1) \delta)=g((m-1) \delta) K(\delta),
$$

as $m \delta \in \mathbb{A}_{+}$. So, summing,

$$
F^{*}(j \delta)=K(\delta) \sum_{m=1}^{j} g((m-1) \delta)
$$

as $F^{*}(0)=K(0)=0$. Note that for $m \leq j$, as $\eta$ is non-decreasing for $\rho \geq 0$,

$$
\Delta_{m}:=m \delta-(m-1) \delta=\delta \eta((m-1) \delta) \leq \delta \eta\left(u_{0}\right)
$$

so that $\Delta_{m} \rightarrow 0$ as $\delta \rightarrow 0$. But as $G^{\prime}(t)=g(t) / \eta(t)$, which is continuous,

$$
\sum_{m=0}^{j-1} g(m \delta) \delta=\sum_{m=0}^{j-1} G^{\prime}(m \delta) \eta(m \delta) \delta=\sum_{m=0}^{j-1} G^{\prime}(m \delta) \Delta_{m} \rightarrow \int_{0}^{u_{0}} G^{\prime}(x) \mathrm{d} x
$$


(for 'Riemann Integral'); likewise for $i$. Next, $G\left(u_{0}\right)>0$, since $g \geq 0$, $g(0)=1$ and $g$ is continuous. Taking limits as $\delta \rightarrow 0$ for $\delta \in \mathbb{A}_{+}$(for which $\left.K(\delta)=F^{*}(\delta)>0\right)$, we then have, as $G\left(u_{0}\right) \neq 0$,

$$
\begin{aligned}
\frac{F^{*}(i \delta)}{F^{*}(j \delta)} & =\frac{K(\delta)}{K(\delta)} \frac{\sum_{m=0}^{i-1} g(m \delta)}{\sum_{m=0}^{j-1} g(m \delta)}=\frac{\sum_{m=0}^{i-1} G^{\prime}(m \delta) \eta(m \delta) \delta}{\sum_{m=0}^{i_{0}-1} G^{\prime}(m \delta) \eta(m \delta) \delta}=\frac{\sum_{m=0}^{i-1} G^{\prime}(m \delta) \Delta_{m}}{\sum_{m=0}^{i_{0}-1} G^{\prime}(m \delta) \Delta_{m}} \\
& \rightarrow \frac{\int_{0}^{u} G^{\prime}(x) \mathrm{d} x}{\int_{0}^{u_{0}} G^{\prime}(x) \mathrm{d} x}=\frac{G(u)}{G\left(u_{0}\right)} .
\end{aligned}
$$

Here, by continuity of $F^{*} \mid \mathbb{A}$ at $u_{0}$,

$$
\lim F^{*}(j \delta)=F^{*}\left(u_{0}\right)=K\left(u_{0}\right)=\kappa\left[1-g\left(u_{0}\right)\right]>0 ;
$$

so

$$
F^{*}(i \delta) \rightarrow G(u) \cdot F^{*}\left(u_{0}\right) / G\left(u_{0}\right) .
$$

Put $c_{1}:=\kappa\left[1-g\left(u_{0}\right)\right] / G\left(u_{0}\right)>0$. Again as $F^{*} \mid \mathbb{A}$ is continuous and $u_{0} \in \mathbb{A}$,

$$
\begin{aligned}
F^{*}(u) & =\lim F^{*}(i \delta)=G(u) \cdot F^{*}\left(u_{0}\right) / G\left(u_{0}\right) \\
& =G(u) K\left(u_{0}\right) / G\left(u_{0}\right)=G(u) \kappa\left[1-g\left(u_{0}\right)\right] / G\left(u_{0}\right)=c_{1} G(u) .
\end{aligned}
$$

So

$$
K(u)=F^{*}(u)=c_{1} G(u) \quad\left(u \in \mathbb{A}_{+}\right) .
$$

We now extend the domain of this equation from $\mathbb{A}_{+}$to the whole of $\mathbb{G}_{+}$, using a key idea due to Goldie (see the Remark below).

Step 2(b). For an arbitrary $u \in \mathbb{G}_{+}$, take $v \in \mathbb{A}_{+}$with $z:=u-{ }_{\rho} v=$ $u \circ v_{\rho}^{-1}>_{\rho} 1_{\rho}$, i.e. with $v<u$ (by Prop. 2). Then

$$
\begin{aligned}
F^{*}(u) & =F^{*}(v+u-v)=F^{*}(v \circ z) \\
& =K(v) g(z)+F^{*}(z) \quad\left(\text { by }(\text { ii }), \text { as } v \in \mathbb{A}_{+} \text {and } z \in \mathbb{R}_{+}\right) \\
& =c_{1} G(v) g(z)+F^{*}(z) \\
& \rightarrow c_{1} G(u) g(0)+0=c_{1} K_{-\gamma}(u) \quad\left(\text { as } z \downarrow 1_{\rho}\right),
\end{aligned}
$$

by continuity of $g$ and $G$, and $F^{*}(0+)=0$.

To derive the form of $g, G, F^{*}$, recall from the beginning of Step 2 that

$$
c_{1} G(u)=F^{*}(u)=K(u)=\kappa[1-g(u)] \quad\left(u \in \mathbb{A}_{+}\right),
$$


with $\kappa>0$. So, by density and continuity on $[0, \infty)$ of $g$,

$$
\kappa[1-g(u)]=c_{1} G(u) \quad\left(u \in \mathbb{G}_{+}\right) .
$$

Thus $g$ is differentiable on $\mathbb{G}_{+}$. Put $\gamma:=c_{1} / \kappa>0$; differentiation yields

$$
-\kappa g^{\prime}(u)=c_{1} g(u) / \eta(u): \quad g^{\prime}(u) / g(u)=-\gamma / \eta(u) \quad\left(u \in \mathbb{G}_{+}\right) .
$$

As $g(0)=1$, if $\rho \in(0, \infty)$

$$
\log g(u)=-\gamma \int_{0}^{u} \frac{\mathrm{d} t}{1+\rho t}=-(\gamma / \rho) \log (1+\rho u): \quad g(u)=(1+\rho u)^{-\gamma / \rho},
$$

as stated in part (a) of the Theorem. The cases $\rho=0$ (where $\eta \equiv 1$ ) and $\rho=\infty$ (where $\eta(t)=t$ ) are similar. From here a routine calculation of $G(u)$ for $u \in \mathbb{G}_{+}$establishes the form of $F^{*}(u)$ as given in part (b) of the Theorem. (For details, see the arXiv version.)

Step 3(a). Here we work in $\mathbb{A}_{-}$where we use pseudo-symmetry. For $\rho \in(0, \infty)$, with $v=-u=u_{\rho}^{-1}$, (inv) gives

$$
1 / g(u)=(1+\rho u)^{\gamma / \rho}=(1+\rho v)^{-\gamma / \rho} .
$$

So

$$
\begin{aligned}
F^{*}(v) & =F^{*}(-u)=-F^{*}(u) / g(u)=-c_{1} K_{-\gamma}(u) / g(u) \\
& =-c_{1}(1+\rho u)^{\gamma / \rho}\left[1-(1+\rho u)^{-\gamma / \rho}\right] / \gamma \\
& =c_{1}\left[1-(1+\rho u)^{\gamma / \rho}\right] / \gamma=c_{1}\left[1-(1+\rho v)^{-\gamma / \rho}\right] / \gamma=c_{1} K_{-\gamma}(v) .
\end{aligned}
$$

The cases $\rho=0$ and $\rho=\infty$ are similar (and simpler): see the arXiv version.

Step 3(b). The final step is to extend the formulas for $K$ and $F^{*}$ from $\mathbb{A}_{-}$ to $\mathbb{G}_{-}$. This is similar to the previous Goldie-inspired argument in Step 2(b), but with $G$ (which is undefined on $\mathbb{G}_{-}$) replaced by $K_{-\gamma}$ using the continuity of $K_{-\gamma}$. (For details, see the arXiv version.)

Remark. Above, we have disaggregated the Goldie proof given in [BinGT, $\S 3.2 .1]$ into three parts. Firstly, we use the integral $G$ of the unknown auxiliary function $g$ (as in [BinO6, Th. 3], albeit here as a Haar integral), where Goldie assumed $g$ explicitly to be the exponential function $e^{\gamma t}$. For Goldie this permits an explicit formula for the corresponding sums (for us the Riemann sums lead to a simple differential equation, which we can solve for $g$, 
giving $G$ ). Secondly, we have partitioned the range of integration by use of a Beck sequence [Bec, Lemma 1.64] (iterating $\circ \delta$ ). Finally, the extension of the relation between $F^{*}$ and $G$ from $\mathbb{A}_{+}$to $\mathbb{G}_{\rho}^{+}$makes explicit a remarkable achievement, due to Goldie (and left implicit in [BinGT, § 3.2.1], our ultimate motivation here): establishment of left-sided continuity from the assumed right-sided continuity " $F^{*}(0+)=0$ ". This overlooked feature was first made explicit in [BinO10] as Theorem 0 there (cf. Th. P1 above), yielding new results, and again put to further extensive use in [BinO11].

We have an immediate Corollary, in which $\sigma>0$, since $g \not \equiv 1$.

Corollary. For $K: \mathbb{G}_{\rho} \rightarrow \mathbb{G}_{\sigma}$ with $\rho \in[0, \infty]$ and $\sigma>0$, the functional equation $\left(B G_{\mathbb{G G}}\right)$ with $K$ for $F^{*}$ may be re-written (with $\gamma$ as there) as

$$
K\left(s \circ_{\rho} t\right)=K(t) \circ_{\gamma} K(s) .
$$

Proof. By Prop. 4, $\mathbb{G}_{\sigma}$ is identified from $\eta_{\sigma}(z)=K_{h}\left(K_{f}^{-1}(z)\right)$ with due attention to orientation. By Th. 4, take $K_{h}=g_{-\gamma}$ and $K_{f}=-K_{-\gamma}$, as then, for $\rho>0, K_{f}\left(\mathbb{G}_{\rho}\right)=(-1 / \gamma, \infty)$ and $K_{f}\left(\mathbb{G}_{\rho}^{+}\right)=(0, \infty)$, and likewise for $\rho=0$. So $\sigma=\gamma$. This may also be justified by direct computation (see the arXiv version).

Armed with the results here, we are now able to freely lift results from [BinO10] concerning when the solution $K_{h f}^{*}: \mathbb{G}_{\eta} \rightarrow \mathbb{G}_{\sigma}$ of $(B G I)$ in fact solves $(B G)$ and so takes the form of a multiple of $K_{\kappa}(u)$ for some $\kappa \in$ $\mathbb{R}$. We recall that in the interests of simplicity we assume that the domain of the asymptotic operator is a subgroup, leaving the reader to refer for results which guarantee this to [BinO7]; we use linear to mean continuous and additive (in the sense of $\S 4$ ) and omit reference to $\varphi$ when context allows.

Theorem 5 (Quantifier-Weakening Theorem, cf. [BinO10, Th. 6], [BinO7, Th. 6]). With $K_{h f}^{*}$ and $\mathbb{A}_{h f}$ as above, suppose that

(i) $\mathbb{A}_{h f}$ is a dense subgroup of $\mathbb{G}_{\eta}$;

(ii) $K_{h f}^{*}$ satisfies the one-sided Heiberg-Seneta boundedness condition

$$
\lim \sup _{u \downarrow 0} K_{h f}^{*}(u) \leq 0 . \quad\left(H S\left(K_{h f}^{*}\right)\right)
$$

Then $\mathbb{A}_{h f}=\mathbb{G}_{\eta}$, for $\eta=\eta^{\varphi}$, and $K_{h f}^{*}$ is linear (continuous and additive):

$$
K_{h f}^{*}(u)=\lim _{x \rightarrow \infty}\left[f\left(x \circ_{\varphi} u\right)-f(x)\right] / h(x)=c K_{\kappa}(u) \quad\left(u \in \mathbb{G}_{\eta}\right)
$$


for some $c, \kappa \in \mathbb{R}$.

Proof of Theorem 5. As we assume here that $\mathbb{A}_{h f}$ is a subgroup, referring to results in [BinO10, Props 3 and 6], $K_{h f}^{*}$ is a finite, subadditive, rightcontinuous extension of the function $G(u):=\lim _{x \rightarrow \infty}\left[f\left(x \circ_{\varphi} u\right)-f(x)\right] / h(x)$ with domain $\mathbb{A}_{h f}$. So $G$ is continuous on $\mathbb{A}_{h f}$, and so $G(a)=K_{\kappa}(a)$, for all $a \in$ $\mathbb{A}_{h f}$ (as in Th. 4). As $\mathbb{A}_{h f}$ is dense, by [BinO10, Prop. 7], $K_{h f}^{*}(u)=K_{\kappa}(u)$ for all $u$. By [BinO10, Prop. 1], $\mathbb{A}_{h f}=\mathbb{G}_{\eta}$ for $\eta=\eta^{\varphi}$ and $K_{h f}^{*}(u)=G(u)$. By Theorem $4, K_{h f}^{*}(u)=c K_{\kappa}(u)$ for some $c, \kappa$.

We turn now to thinnings of the condition $(H S)$ of Theorem 5. For this we need some definitions from [BinO10].

Definitions. 1. In a normed group $G$ (i.e. topologized by a group norm), say that $\Sigma \subseteq G$ is locally Steinhaus-Weil $(S W)$, or has the $S W$ property locally, if for $x, y \in \Sigma$ and, for all $\delta>0$ sufficiently small, the sets

$$
\Sigma_{z}^{\delta}:=\Sigma \cap B_{\delta}(z),
$$

for $z=x, y$, have the interior-point property: that $\Sigma_{x}^{\delta} \pm \Sigma_{y}^{\delta}$ has $x \pm y$ in its interior. (Here, as before, $B_{\delta}(x)$ is the open ball about $x$ of radius $\delta$.) See [BinO3] for conditions under which this property is implied by the interior-point property of the sets $\Sigma_{x}^{\delta}-\Sigma_{x}^{\delta}$ (cf. [BarFN]); see also the 'rich list' of examples in [BinO11, §4.5] (or the arXiv version), which are used in [BinO8,10,11,13,14], [MilMO].

2. For $G$ again a normed group, say that $\Sigma \subseteq G$ is shift-compact if for each null sequence $\left\{z_{n}\right\}$ (i.e. with $z_{n} \rightarrow 1_{G}$ ) there are $t \in \Sigma$ and an infinite $\mathbb{M} \subseteq \mathbb{N}$ such that

$$
\left\{t+z_{m}: m \in \mathbb{M}\right\} \subseteq \Sigma .
$$

See [BinO4], and for the group-action aspects, [MilO].

We can now state some thinned variants of Th. 5 .

Theorem 6 (Thinned Quantifier Weakening Theorem; [BinO10, Th. $1^{\prime}$ ], cf. [BinO7, $\S 6$ Th. 5]). Theorem 5 above holds with condition (ii) replaced by any one of the following:

(ii-a) $K_{h f}^{*}$ satisfies the Heiberg-Seneta boundedness condition thinned out to a symmetric set $\Sigma$ that is locally $S W$, i.e.

$$
\lim \sup _{u \rightarrow 0, u \in \Sigma} K_{h f}^{*}(u) \leq 0 ;
$$


(ii-b) $K_{h f}^{*}$ is linearly bounded above on a locally $S W$ subset $\Sigma \subseteq \mathbb{G}_{\eta}^{+}$accumulating at $1_{\eta}$, so that in particular

$$
\lim \sup _{u \downarrow 0, u \in \Sigma} K_{h f}^{*}(u) \leq 0 ;
$$

(ii-c) $K_{h f}^{*}$ is bounded above on a locally $S W$ subset $\Sigma \subseteq \mathbb{A}_{+}:=\mathbb{A} \cap \mathbb{G}_{\eta}^{+}$ accumulating at 0 with $\mathbb{A}$ as below, that is, the following limsup is finite:

$$
\lim \sup _{u \downarrow 0, u \in \Sigma} K_{h f}^{*}(u)<\infty ; \quad\left(S W-H S\left(K_{h f}^{*}\right)\right)
$$

(ii-d) $S$ is bounded on a subset $\Sigma \subseteq \mathbb{A}$ that is shift-compact (e.g. on a set that is locally $S W$, and so on an open set) and

$\mathbb{A}=\mathbb{A}_{h f}:=\left\{u: K_{h f}(u):=\lim _{x \rightarrow \infty}\left[f\left(x \circ_{\varphi} u\right)-f(x)\right] / h(x)\right.$ exists and is finite $\}$.

Proof. This follows from the Popa variant of [BinO10, Theorem 1'], the proof of which follows from Theorems G2 and G3 of $\S 4$ above in place of [BinO10, Theorems $0^{\prime}$ and 0].

The classical Quantifier Weakening Theorems of regular variation ([BinGT, $\S 1.4 .3$ and $\S 3.2 .5]$ ) are re-stated below as Theorems $\mathrm{K}$ and BKdH. There, one needs as side-condition the Heiberg-Seneta condition $H S$ restated multiplicatively here as (limsup) (or a thinned version of it, as in Theorem 6). Recall from above the ${ }^{*}$ notation (as in $g^{*}$ ) signifying that limsup replaces lim .

Theorem $\mathbf{K}$ (cf. [BinGT, Th. 1.4.3] replacing $\rho$ there with $\gamma$ as in Theorem 4). Suppose that

$$
\lim \sup _{\lambda \downarrow 1} K_{f}^{*}(\lambda) \leq 1 . \quad(\limsup -f)
$$

Then the following are equivalent:

(i) there exists $\gamma \in \mathbb{R}$ such that

$$
f(\lambda x) / f(x) \rightarrow \lambda^{\gamma} \quad(x \rightarrow \infty)(\forall \lambda>0) ;
$$

(ii) $g(\lambda)=\lim _{x \rightarrow \infty} f(\lambda x) / f(x)$ exists, finite for all $\lambda$ in a Baire/measurable non-negligible subset of $(0, \infty)$;

(iii) $g(\lambda)$ exists, finite, for all $\lambda$ in a dense subset of $(0, \infty)$;

(iv) $g(\lambda)$ exists, finite for $\lambda=\lambda_{1}, \lambda_{2} \in(0, \infty)$ with $\left(\log \lambda_{1}\right) / \log \lambda_{2}$ irrational.

Theorem $\mathrm{K}$ is an immediate corollary of Theorem 5 with $\rho=\infty$, as (limsup- $f$ ) iff $\left(H S\left(K_{f}^{*}\right)\right)$. The final assertion follows from Kronecker's theorem [HarW, Ch. 23]. 
Theorem BKdH (cf. [BinGT, Th. 3.2.5]). For $h$ with

$$
\lim _{x \rightarrow \infty} h(\lambda x) / h(x)=\lambda^{\gamma} \quad(\lambda>0),
$$

and

$$
\lim \sup _{\lambda \downarrow 1} K_{h f}^{*}(\lambda) \leq 0, \quad \quad(\limsup -h f)
$$

the following are equivalent:

(i) $K_{h f}(\lambda):=\lim _{x \rightarrow \infty}[f(\lambda x)-f(x)] / h(x)$ exists, finite for all $\lambda>0$, and $K_{h f}(\lambda)=c \eta_{\rho}^{-1}\left(\lambda^{\rho}\right)$ for some $c$ and all $\lambda$ on a Baire/measurable non-negligible subset of $(0, \infty)$;

(ii) $K_{h f}(\lambda)$ exists, finite for all $\lambda$ in a non-negligible subset of $(0, \infty)$;

(iii) $K_{h f}(\lambda)$ exists, finite, for all $\lambda$ in a dense subset of $(0, \infty)$;

(iv) $K_{h f}(\lambda)$ exists, finite for $\lambda=\lambda_{1}, \lambda_{2} \in(0, \infty)$ with $\left(\log \lambda_{1}\right) / \log \lambda_{2}$ irrational.

Theorem BKdH is an immediate corollary of the case $\rho=\infty$ in Theorem 4. As before the final assertion follows from Kronecker's theorem.

The motivation for this paper was the treatment of Theorems $\mathrm{K}$ and BKdH above via Popa groups in [BinO7, §7] (specifically $(G F E)$ and $(G S)$ there and their equivalence), using the extra power of the extra generality here to provide a unified and simplified treatment.

\section{Complements}

1. Beurling's Tauberian theorem. To extend the Wiener Tauberian Theorem (Theorem W, say) Beurling introduced (in unpublished lectures of 1957) his Tauberian Theorem (below), extending Theorem $\mathrm{W}$ from convolutions to 'convolution-like' operations. We need the Beurling convolution:

$$
F *_{\varphi} H(x):=\int_{-\infty}^{+\infty} F\left(\frac{x-u}{\varphi(x)}\right) H(u) \frac{\mathrm{d} u}{\varphi(x)}=\int F(-t) H\left(x \circ_{\varphi} t\right) \mathrm{d} t .
$$

This is an asymptotic version, involving the function $\eta_{x}($.$) of \S 3$ :

$$
\eta_{x}(t):=\varphi\left(x \circ_{\varphi} t\right) / \varphi(x)
$$

of an ordinary convolution (below). 
Theorem B (Beurling's Tauberian theorem). For $K \in L_{1}(\mathbb{R})$ with Fourier transform $\hat{K}$ non-zero on $\mathbb{R}$, and $\varphi$ Beurling slowly varying, that is

$$
\eta_{x}(t) \rightarrow 1, \quad(x \rightarrow \infty) \quad(t \geq 0):
$$

if $H$ is bounded, and

$$
K *_{\varphi} H(x) \rightarrow c \int K(y) \mathrm{d} y,
$$

then for all $F \in L_{1}(\mathbb{R})$

$$
F *_{\varphi} H(x) \rightarrow c \int F(y) \mathrm{d} y \quad(x \rightarrow \infty) .
$$

This reduces to Theorem $\mathrm{W}$ on replacing $\varphi$ by 1 . For an elegant proof, see [Kor, IV.11].

In Theorem $\mathrm{W}$, the argument in the integral above (with $\varphi=1$ ) is $x-$ $u$, and so is a convolution (written additively, or $x / u$ multiplicatively). In Theorem B, the integral is merely 'convolution-like'. Beurling was able to use his form of slow variation, $(B S V)$, to reduce easily to convolution form, and so to Theorem W. His motivation was the Tauberian theorem for the Borel summability method, important in summability theory, complex analysis and probability [Kor, VI]. For applications in probability, see e.g. [Bin1,2].

Beurling convolution is an asymptotic convolution: to within a factor $\eta_{x}(t) \rightarrow 1$, it is the proper convolution

$$
\left(f *_{\varphi} g\right)(x):=\int_{\mathbb{G}_{\rho}} f\left(-t / \eta_{x}(t)\right) g\left(x \circ \circ_{\varphi} t\right) \mathrm{d} \eta_{\mathbb{G}_{\rho}}(t) \quad\left(x \in \mathbb{G}_{\rho}\right) .
$$

For, given $x$ and $t$, solving for $s$ the equation

$$
x=\left(x \circ_{\varphi} t\right) \circ_{\varphi} s=x+t \varphi(x)+s \varphi(x+t \varphi(x))
$$

yields

$$
s=-t \varphi(x) / \varphi(x+t \varphi(x))=-t / \eta_{x}(t)
$$

as the 'inverse of $t$ ' (relative to the binary operation $\circ_{\varphi}$ acting on the set $\mathbb{G}_{\rho}$, i.e. the domain of the group).

For $\varphi \in S E$, the corresponding asymptotic convolution is

$$
\left(f *_{\varphi} g\right)(x):=\int f\left(-t / \eta_{\rho}(t)\right) g\left(x \circ_{\varphi} t\right) \mathrm{d} \eta_{\mathbb{G}_{\rho}}(t) .
$$


For $\varphi(x):=\eta_{\rho}(x) \in G S$, since

$$
\eta_{x}(t):=\frac{\varphi(x+t \varphi(x))}{\varphi(x)}=\frac{\eta_{\rho}\left(x+t \eta_{\rho}(x)\right)}{\eta_{\rho}(x)}=\eta_{\rho}(t),
$$

$(f * \varphi g)(x)$ becomes

$$
\left(f *_{\eta_{\rho}} g\right)(x):=\int f\left(-t / \eta_{\rho}(t)\right) g\left(x \circ_{\eta_{\rho}} t\right) \mathrm{d} \eta_{G_{\rho}}(t)=\int f\left(-{ }_{\rho} t\right) g\left(x+{ }_{\rho} t\right) \mathrm{d} \eta_{\mathbb{G}_{\rho}}(t),
$$

with the notation of Theorem 2. So in this case the asymptotic convolution becomes ordinary convolution for the Popa group $\left(\mathbb{G}_{\rho}, \circ_{\rho}\right)$.

2. Triple Encoding. We encounter here the unusual situation of three different mathematical structures occurring together, each of which encodes the other two, in the sense that the other two can be obtained from it.

2.1 'Uniqueness' of the topology: the Converse Haar Theorem. Weil [Wei] viewed his retrieval of the topological-group structure from the measurealgebra structure [Fre] as encoded by the Haar-measurable subsets (cf. [Kod]) to be the converse to Haar's theorem on the existence of an invariant measure. (Here one may work either, following Weil, to within a dense embedding in a locally compact group (as in [BinO13, Th. 7M, Remark]), or, following Mackey, uniquely up to homeomorphism, granted the further assumption of an analytic Borel structure [Mac, Th. 7.1]. For more on this see [BinO13, §8.16].) In fact, here the measure structure is already encoded by the density topology $\mathcal{D}$ via the Haar density theorem, for which see [Mue], [Hal, §61(5), p. 268], [Oxt, Ch. 22]; cf. [BinO2, §7; Th. 6.10], [BinO9]. This view is partially implicit in [Amb], where refinement of one invariant measure $\mu_{1}$ by another $\mu_{2}$ holds when sets in $\mathcal{M}_{+}\left(\mu_{2}\right)$ contain sets in $\mathcal{M}_{+}\left(\mu_{1}\right)$ (as in the refinement of one topology by another, with $\mathcal{M}_{+}(\mu)$ the $\mu$-measurable subsets of finite positive $\mu$-measure). This falls within the broader aim of retrieving a topological group structure from a given (one-sidedly) invariant topology $\tau$ on a group $G$, when $\tau$ arises from refinement of a topological group structure (i.e. starting from a semitopological group structure $(G, \tau)$ ). Also relevant here are Converse Steinhaus-Weil results, as in [BinO13, §2 Prop. $7, \S 4])$.

2.2 'Uniqueness' of the metric. We have mentioned the Birkhoff-Kakutani (metrization) theorem on the existence of a left-invariant metric in a firstcountable topological group, which we view as a (group-)normability theorem: see [Ost2, §2.1]. Struble's theorem ([Str]; [DieS Ch. 8]) identifies the 
group norm directly from the left-invariant Haar measure $\eta_{G}$ on the locally compact group $G$ and its topology via Weil-norms [BinO13, §6]

$$
\|x\|:=\sup _{n}\left\{\eta_{G}\left(x U_{n} \triangle U_{n}\right)\right\}
$$

with $\triangle$ denoting symmetric difference and $\left\{U_{n}\right\}_{n \in \mathbb{N}}$ a descending sequence of open sets (each with compact closure) giving an open neighbourhood basis at the identity $1_{G}$. (It is of interest that for any such norm, as one might expect from here, isometric subsets of $G$ have identical $\eta_{G}$ measure [Ban], [DieS, Th. 82].) When the norm generating the topology is bi-invariant, Klee's completeness theorem [Kle], [DieS, Th. 8.16] asserts that if the topology is completely metrizable, then in fact the norm is itself complete.

2.3 'Uniqueness' of the group structure. We confine ourselves here to one example, the Popa context above with $\rho=1$. Here the corresponding norm $\|x\|:=|\log (1+x)|$ identifies the group structure $\circ_{1}$ as unique. For, if - denotes an abelian group operation consistent with the norm distance $d(x, y)=\left\|y^{-1} x\right\|$, in the sense that for $c>0$

$$
\|c\|=d\left(1_{G}, c\right)=\log (1+c)
$$

then for $a \geq b>0$, writing $c=a \cdot b^{-1}$ so that $a=b \cdot c$,

$$
\left\|a \cdot b^{-1}\right\|=d(a, b)=\log (1+a)-\log (1+b)=\log (1+a) /(1+b) .
$$

So, since $\|c\|=\left\|a \cdot b^{-1}\right\|$,

$\log (1+c)=\log (1+a) /(1+b): \quad b \cdot c=a=(1+b)(1+c)-1=b+c+b c$

that is $b \cdot c=b \circ_{1} c$. (In particular, this clarifies the connection with the invariance of the 'continued-fraction transformation' $x \mapsto 1 / x-[1 / x]$ in the Gauss-Kuzmin theorem under Gauss's measure - cf. Remark 3 in $\S 2$.).

2.4 Higher dimensions. One can work more generally in higher dimensions (finite or infinite). We refer for detail here to [BinO15,16].

Postscript. The whole area of regular variation stems from the pioneering work of Jovan Karamata (1902-1967) in 1930. The present paper stems from his work with Ranko Bojanic (1925-2017) of 1963 [BojK]. The first author offers here a reminiscence of his first meeting with Ranko Bojanic (in 1988, over dinner, at a conference at Ohio State University, Columbus OH). He asked Professor Bojanic why he and Karamata had stopped their work on 
regular variation in 1963. He replied unhesitatingly 'Because we didn't know what it was good for'. Analysts in general, and probabilists in particular, do now know what it is good for. Our aim here has been to demonstrate the power, and ongoing influence, of their work, with the benefit of 56 years worth of hindsight.

Acknowledments. We are most grateful to the Referee for his careful, thoughtful and constructive comments, which have helped to improve this paper significantly. Our indebtedness to (and admiration of) Charles Goldie's insights in [BinG1], the ultimate source of the current paper, are of equal measure.

\section{References}

[AczD] J. Aczél and J. Dhombres, Functional equations in several variables. Encycl. Math. App. 31, Cambridge University Press, 1989.

[Amb] W. Ambrose, Measures on locally compact topological groups. Trans. Amer. Math. Soc. 61 (1947), 106-121.

[ArhT] A. Arhangel'skii and M. Tkachenko, Topological groups and related structures. World Scientific, 2008.

[Arr] M. El Arrouchi, Characterization of tail distributions based on record values by using Beurling's Tauberian theorem. Extremes 20 (2017), 111-120. [Ban] C. Bandt, Metric invariance of Haar measure, Proc. Amer. Math. Soc. 87 (1983), 65-69.

[BarAE] H. M. Barakat and M. A. Abd Elgawad, Asymptotic behavior of the joint record values, with applications. Statist. Probab. Lett. 124 (2017), $13-21$.

[BarFN] A. Bartoszewicz, M. Filipczak and T. Natkaniec, On Smítal properties. Topology Appl. 158 (2011), 2066-2075.

[Bec] A. Beck, Continuous flows on the plane, Grundl. math. Wiss. 201, Springer, 1974.

[Bil] P. Billingsley, Ergodic theory and information. Wiley, 1965.

[Bin1] N. H. Bingham, Tauberian theorems and the central limit theorem. Ann. Prob. 19 (1981), 221-231.

[Bin2] N. H. Bingham, Riesz means and Beurling moving averages, Risk and Stochastics (Ragnar Norberg Memorial volume, ed. P. M. Barrieu) Imperial College Press, 2019, Ch. 8, 159-172 (arXiv:1502.07494).

[BinG1] N. H. Bingham and C. M. Goldie, Extensions of regular variation. I. Uniformity and quantifiers, Proc. London Math. Soc. 44 (1982), 473-496. 
[BinG2] N. H. Bingham and C. M. Goldie, On one-sided Tauberian conditions, Analysis 3 (1983), 159-188.

[BinGT] N. H. Bingham, C. M. Goldie and J. L. Teugels, Regular variation, 2nd ed., Cambridge University Press, 1989 (1st ed. 1987).

[BinO1] N. H. Bingham and A. J. Ostaszewski, Beyond Lebesgue and Baire II: bitopology and measure-category duality. Colloq. Math. 121 (2010), no. 2, 225-238.

[BinO2] N. H. Bingham and A. J. Ostaszewski, Normed versus topological groups: dichotomy and duality. Dissert. Math. 472 (2010), 138 pp.

[BinO3] N. H. Bingham and A. J. Ostaszewski, Regular variation without limits, J. Math. Anal. Appl. 370 (2010), 322-338.

[BinO4] N. H. Bingham and A. J. Ostaszewski, Dichotomy and infinite combinatorics: the theorems of Steinhaus and Ostrowski. Math. Proc. Cambridge Philos. Soc. 150 (2011), 1-22.

[BinO5] N. H. Bingham and A. J. Ostaszewski, Beurling slow and regular variation, Trans. London Math. Soc., 1 (2014) 29-56.

[BinO6] N. H. Bingham and A. J. Ostaszewski, Cauchy's functional equation and extensions: Goldie's equation and inequality, the Gołąb-Schinzel equation and Beurling's equation. Aequationes Math. 89 (2015), 1293-1310.

[BinO7] N. H. Bingham and A. J. Ostaszewski, Beurling moving averages and approximate homomorphisms, Indag. Math. 27 (2016), 601-633 (fuller version: arXiv1407.4093).

[BinO8] N. H. Bingham and A. J. Ostaszewski, Category-measure duality: convexity, midpoint convexity and Berz sublinearity. Aequationes Math. 91 (2017), 801-836.

[BinO9] N. H. Bingham and A. J. Ostaszewski, Beyond Lebesgue and Baire IV: Density topologies and a converse Steinhaus-Weil theorem, Topology Appl., 239 (2018), 274-292 (arXiv1607.00031).

[BinO10] N. H. Bingham and A. J. Ostaszewski, Additivity, subadditivity and linearity: Automatic continuity and quantifier weakening. Indag. Math. (N.S.) 29 (2018), 687-713 (arXiv 1405.3948v3).

[BinO11] N. H. Bingham and A. J. Ostaszewski, Variants on the Berz sublinearity theorem, Aequationes Math. 93 (2019), 351-369 [arXiv:1712.05183].

[BinO12] N. H. Bingham and A. J. Ostaszewski, Set theory and the analyst, European. J. Math. 5 (2019), 2-48 [arXiv:1801.09149].

[BinO13] N. H. Bingham and A. J. Ostaszewski, The Steinhaus-Weil property: its converse, Solecki amenability and subcontinuity, arXiv:1607.00049v3. [BinO14] N. H. Bingham and A. J. Ostaszewski, Sequential regular variation: 
extensions of Kendall's theorem, arXiv:1901.07060.

[BinO15] N. H. Bingham and A. J. Ostaszewski, Multivariate general regular variation: Popa groups on vector spaces, arXiv:1910.05816.

[BinO16] N. H. Bingham and A. J. Ostaszewski, Multivariate Popa groups and the Goldie Equation,arXiv:1910.05817.

[Bir] G. Birkhoff, A note on topological groups. Compos. Math. 3 (1936), 427-430.

[BojK] R. Bojanić and J. Karamata, On a class of functions of regular asymptotic behavior, Math. Research Center Tech. Report 436, Madison, Wis. 1963; reprinted in Selected papers of Jovan Karamata (ed. V. Marić, Zevod za Udžbenike, Beograd, 2009), 545-569.

[Bra] J. Braconnier, Spectres d'espaces et de groupes topologiques. Portugaliae Math. 7 (1948), 93-111.

[Brz1] J. Brzdęk, The Gołąb-Schinzel equation and its generalizations, Aequat. Math. 70 (2005), 14-24.

[Brz2] J. Brzdęk, Subgroups of the group $\mathbb{Z}_{n}$ and a generalization of the Gołąb-Schinzel functional equation, Aequat. Math. 43 (1992), 59-71.

[BrzM] J. Brzdęk and A. Mureńko, On a conditional Gołąb-Schinzel equation, Arch. Math. 84 (2005), 503-511.

[Chu1] J. Chudziak, Semigroup-valued solutions of the Gołąb-Schinzel type functional equation, Abh. Math. Sem. Univ. Hamburg, 76 (2006), 91-98.

[Chu2] J. Chudziak, Semigroup-valued solutions of some composite equations, Aequat. Math. 88 (2014), 183-198.

[Coh] P. M. Cohn, Algebra, vol. 1, 2 $2^{\text {nd }}$ ed. Wiley, New York, 1982 (1st edn 1974).

[DieS] J. Diestel and A. Spalsbury, The joys of Haar measure. Graduate Studies in Mathematics 150, Amer. Math. Soc., 2014.

[Ell] R. Ellis, Lectures on topological dynamics. W. A. Benjamin, 1969.

[EllE] D. B. Ellis and R. Ellis, Automorphisms and equivalence relations in topological dynamics. London Math. Soc. Lect. Note Series 412. Cambridge University Press, 2014.

[Eng] R. Engelking, General topology, $2^{\text {nd }}$ ed., Heldermann, $1989\left(1^{\text {st }}\right.$ ed 1977).

[FalKCP1] M. Falk, A. Khorrami Chokami, and S. A. Padoan, Some results on joint record events. Statist. Probab. Lett. 135 (2018), 11-19.

[FalKCP2] M. Falk, A. Khorrami Chokami, and S. A. Padoan, On multivariate records from random vectors with independent components. J. Appl. Probab. 55 (2018), 43-53. 
[Fre] D. Fremlin, Measure theory Vol. 3: Measure algebras. Corrected $2^{\text {nd }}$ printing of the 2002 original. Torres Fremlin, Colchester, 2004.

[dHa] L. de Haan, Equivalence classes of regularly varying functions. Stochastic Processes Appl. 2 (1974), 243-259.

[Hal] P. R. Halmos, Measure theory, Grad. Texts in Math. 18, Springer 1974 ( $1^{\text {st }}$ ed. Van Nostrand, 1950).

[HarW] G. H. Hardy and E. M. Wright, An introduction to the theory of numbers. $6^{\text {th }}$ ed. Revised by D. R. Heath-Brown and J. H. Silverman. With a foreword by Andrew Wiles. Oxford University Press, 2008.

[Haz] M. Hazewinkel, Formal groups and applications. AMS Chelsea Publishing, 2012 (corrected reprint of the 1978 original).

[HewR] E. Hewitt and K. A. Ross, Abstract harmonic analysis, Vol. I, Grundl. math. Wiss. 115, Springer 1963 [Vol. II, Grundl. 152, 1970].

[HilP] E. Hille and R. S. Phillips, Functional Analysis and Semi-groups, $3^{\text {rd }}$ ed., AMS Colloquium Publications XXXI, 1974 (1 ${ }^{\text {st }}$ ed., 1957).

[Jab1] E. Jabłońska, Continuous on rays solutions of an equation of the Gołąb-Schinzel type. J. Math. Anal. Appl. 375 (2011), 223-229.

[Jab2] E. Jabłońska, On locally bounded above solutions of an equation of the Gołąb-Schinzel type. Aequat. Math. 87 (2014),125-133.

[Jab3] E. Jabłońska, On solutions of some generalizations of the GołąbSchinzel equation, in: Functional equations in mathematical analysis, ed. J. Brzdęk and Th. M. Rassias, Springer Optim. Appl. 52 (2012), 509-521.

[Jac] N. Jacobson, Lectures in Abstract Algebra, vol. I. Van Nostrand, New York, 1951.

[Jav] P. Javor, On the general solution of the functional equation $f(x+$ y $f(x))=f(x) f(y)$. Aequat. Math. 1 (1968), 235-238.

[Kak1] S. Kakutani, Über die Metrisation der topologischen Gruppen, Proc. Imp. Acad. Tokyo 12 (1936), 82-84 (reprinted in [Kak2]).

[Kak2] S. Kakutani, Selected papers. Vol. 1. (Ed. R. R. Kallman), Contemporary Mathematicians. Birkhäuser, 1986.

[Kle] V. Klee, Invariant extensions of linear functionals, Pacific J. Math. 4 (1954), 37-46.

[Kod] K. Kodaira, Uber die Beziehung zwischen den Massen und den Topologien in einer Gruppe. Proc. Phys.-Math. Soc. Japan (3) 23 (1941), 67-119. [Kor] J. Korevaar, Tauberian theorems: A century of development. Grundl. math. Wiss. 329, Springer, 2004.

[Kuc] Marek Kuczma, An introduction to the theory of functional equations and inequalities. Cauchy's equation and Jensen's inequality. 2nd ed., 
Birkhäuser, 2009 [1st ed. PWN, Warszawa, 1985].

[LanS] H. Lanzinger and U. Stadtmüller,Tauberian theorems and limit distributions for upper order statistics. Dédié à Jovan Karamata. Publ. Inst. Math. (N.S.) 71(85) (2002), 41-53.

[Loo] L. H. Loomis, An introduction to abstract harmonic analysis. Van Nostrand, 1953.

[Mac] G. W. Mackey, Borel structure in groups and their duals. Trans. Amer. Math. Soc. 85 (1957), 134-165.

[MilMO] H. I. Miller, L. Miller-van Wieren, and A. J. Ostaszewki, Beyond Erdős-Kunen-Mauldin: Singular sets with shift-compactness properties, arXiv:1901.09654.

[MilO] H. I. Miller and A. J. Ostaszewski, Group action and shift-compactness, J. Math. Anal. App. 392 (2012), 23-39.

[Mue] B. J. Mueller, Three results for locally compact groups connected with the Haar measure density theorem. Proc. Amer. Math. Soc. 16 (6) (1965), 1414-1416.

[Ost1] A. J. Ostaszewski, Regular variation, topological dynamics, and the uniform boundedness theorem. Topology Proc. 36 (2010), 305-336.

[Ost2] A. J. Ostaszewski, Beyond Lebesgue and Baire III: Steinhaus' theorem and its descendants. Topology Appl. 160 (2013), 1144-1154.

[Ost3] A. J. Ostaszewski, Beurling regular variation, Bloom dichotomy, and the Gołąb-Schinzel functional equation, Aequat. Math. 89 (2015), 725-744.

[Ost4] A. J. Ostaszewski, Homomorphisms from Functional Equations: The Goldie Equation, Aequat. Math. 90 (2016), 427-448 (arXiv: 1407.4089).

[Ost5] A. J. Ostaszewski, Homomorphisms from Functional Equations in Probability, in: Developments in Functional Equations and Related Topics, ed. J. Brzdęk et al, Springer Optim. Appl. 124 (2017), 171-213.

[Oxt] J. C. Oxtoby: Measure and category, 2nd ed. Graduate Texts in Math. 2, Springer, 1980.

[PalW] R. E. A. C. Paley and N. Wiener, Fourier transforms in the complex domain. Amer. Math. Soc. Colloquium Publ. 19, 1934.

[Pet] B. J. Pettis, On continuity and openness of homomorphisms in topological groups. Ann. of Math. (2) 52 (1950), 293-308.

[Pic] S. Piccard, Sur les ensembles de distances des ensembles de points d'un espace Euclidien. Mém. Univ. Neuchâtel 13, 212 pp. 1939.

[Pop] C. G. Popa, Sur l'équation fonctionelle $f[x+y f(x)]=f(x) f(y)$, Ann. Polon. Math. 17 (1965), 193-198.

[Rud] W. Rudin, Fourier analysis on groups. Wiley, 1962. 
[Ste] H. Steinhaus, Sur les distances des points de mesure positive. Fund. Math. 1 (1920), 83-104.

[Str] R. Struble, Metrics in locally compact groups. Compositio Math. 28 (1974), 217-222.

[Tit] E. C. Titchmarsh, Introduction to the theory of Fourier integrals. $3^{\text {rd }}$ ed. Chelsea, 1986 ( $1^{\text {st }}$ ed. Oxford Univ Press, 1937).

[Wei] A. Weil, L'intégration dans les groupes topologiques, Actualités Scientifiques et Industrielles 1145, Hermann, 1965 ( $1^{\text {st }}$ ed. 1940).

[Wie] N. Wiener, The Fourier integral and certain of its applications. Reprint of the 1933 edition (foreword by Jean-Pierre Kahane), Cambridge University Press, 1988

Mathematics Department, Imperial College, London SW7 2AZ; n.bingham@ic.ac.uk Mathematics Department, London School of Economics, Houghton Street, London WC2A 2AE; A.J.Ostaszewski@lse.ac.uk 